\title{
Dynamic Motion Planning of 3D Human Locomotion Using Gradient-based Optimization
}

\author{
Hyung Joo Kim, Qian Wang, Salam Rahmatalla, Colby C. Swan`, Jasbir S. Arora, \\ Karim Abdel- Malek and Jose G. Assouline \\ Center for Computer Aided Design \\ College of Engineering \\ The University of lowa \\ lowa City, lowa 52242 U.S.A.
}

\begin{abstract}
Since humans can walk with an infinite variety of postures and limb movements, there is no unique solution to the modeling problem to predict human gait motions. Accordingly, we test herein the hypothesis that the redundancy of human walking mechanisms makes solving for human joint profiles and force time histories an indeterminate problem best solved by inverse dynamics and optimization methods. A new optimization-based human-modeling framework is thus described for predicting three dimensional human gait motions on level and inclined planes. The basic unknowns in the framework are the joint motion time-histories of a 25-degree of freedom human model and its six global degrees of freedom. The joint motion histories are calculated by minimizing an objective function such as deviation of the trunk from upright posture that relates to the human model's performance. A variety of important constraints are imposed on the optimization problem, including: (1) satisfaction of dynamic equilibrium equations by requiring the model's zero moment point (ZMP) to lie within the instantaneous geometrical base of support; (2) foot collision avoidance; (3) limits on ground-foot friction; and (4) vanishing yawing moment. Analytical forms of objective and constraint functions are presented and discussed for the proposed human modeling framework in which the resulting optimization problems are solved using gradient-based mathematical programming techniques. When the framework is applied to modeling of bipedal locomotion on level and inclined planes, acyclic human walking motions are obtained that are smooth and realistic as opposed to less natural robotic motions. Aspects of the modeling framework requiring further investigation and refinement are discussed as well as potential applications of the framework in biomechanics.
\end{abstract}

\section{INTRODUCTION}

\subsection{PREVIOUS WORK}

\footnotetext{
- Corresponding author. E-mail: colby-swan@uiowa.edu; Surface mail: Dept. of Civil \& Environmental Engineering, 4120 Seamans Center, The University of lowa, lowa City, lowa 52242 U.S.A.
} 
Normal human locomotion requires complex control between multiple limb and body segments working in synchronization to provide the most shock-absorbing and energy-efficient forward movement possible (Saunders et al, 1953). Within the realm of normal locomotion, specific gait characteristics arise due to variations in muscle strength, dynamic ranges of motion, the relative shape, position and function of neuromuscular and musculoskeletal structures, and ligamentous and capsular constraints on the joints. Although human locomotion has been the subject of intense research (Saunders et al., 1953; Perry, 1992; Sutherland et al, 1988, 1994; Skinner, 2003), many aspects remain enigmatic. While the existing technologies of motion capture and gait analysis can measure and document fine details of human locomotion, they do not have predictive capabilities which are needed to more fully understand how different anthropometries and states of health or disease affect human locomotion. Toward this end a predictive framework for modeling the dynamics of human walking is put forward in this article.

Digital human modeling is a relatively new field that includes development of mathematical models for full-body kinematics and dynamics. This field has gained considerable momentum in recent years with support from manufacturing industries who seek to forestall the need for expensive prototyping and human testing of their products. Instead their products can be tested with virtual humans which are mathematical models that represent human anthropometry, kinematics, and dynamics. Development of mathematically based virtual humans is known as digital human modeling. While this field has a tremendous range of applications in the manufacturing and evaluation of consumer products, we believe it also has an equally broad range of biomedical applications. In this paper, we focus attention on the challenge of controlling a digital human model so that it will walk in a way that realistically emulates normal human locomotion.

Predicting natural bipedal gait motion for a digital human model with specified anthropometric parameters can involve solution of non-trivial dynamics problems in which joint angle rotations, joint torque profiles, and ground reaction forces are all unknowns. Many of the physical constraints on human locomotion, such as joint angle limits, joint torque limits, and lack of ground penetration are typically specified as inequality constraints. Since it is not known a priori whether or not these constraints will be active at any given instant, they contribute to the difficulty in solving the dynamics problem.

Due to the indeterminacy of the dynamics problem associated with human locomotion, several different approaches to modeling of human locomotion have been described in the literature. For simplicity, these approaches can be categorized into those that solve the dynamics problem of human motion either directly or indirectly, and those that do not solve the dynamics problem at all and deal strictly with the kinematics. Statistical database methods, for example, deal strictly with kinematics and rely on searching through pre-established motion databases and finding appropriate walking motions that are then sequenced and scaled to correspond with the anthropometry of the digital human model under consideration (Furusho and Masubuchi 1986; Faraway et al. 1999). As such methods do not involve solving the dynamics equations of motion 
they do not yield joint forces or the related work that they do. For this reason, such methods cannot evaluate energy expenditure or the stability of different walking motions, or even adapt to walking on sloped inclines. Such methods may also provide little insight on the subtleties of human locomotion.

There is a considerable variation among the motion planning methods that address the dynamics of human locomotion. To better understand the preceding works in this area, it is helpful to distinguish between those directed at motion planning of robots, and those directed at modeling realistic human walking behaviors. It is also useful to further distinguish between those methods that solve the dynamics problem directly, and those that solve the dynamic problem in an inverse manner.

\subsubsection{Robotic vs. human motion}

In robotics, fast solution of the dynamics problem is needed to facilitate real-time control. Accordingly, large numbers of artificial equality constraints are imposed on the robotic gait parameters by a priori specification of hip/limb motions or by specifying trajectories in time of the zero moment point (ZMP) ${ }^{\star}$. Although such artificial constraints speed up the solution process by reducing the feasible domain, they can be overly restrictive to the point where the resulting motions may be unsmooth, unnatural, and unable to adapt to changes in the mission goals, the anthropometric data, or the terrain conditions. Among the works from this category, Azevedo et al. (2002) synthesized a gait motion off-line and applied a simple law to control a real system. In the off-line motion synthesis, equality constraints on the initial, final, and intermediate positions of the ankle, toe, and hip joints were imposed to ensure obstacle avoidance, static stability, and symmetry of gait stages. Nishiwaki and Kagami (2002) proposed a real-time walking pattern generator to make a humanoid follow specified foot locations on the ground and to maintain a desired zero moment point trajectory. Kuffner et al. (2002) employed a randomized search strategy based on "Rapidly-exploring Random Trees" (RRTs) to find dynamically stable trajectories. In this approach, a collision-free, statically stable, kinematical path is first determined and then iteratively adjusted to become a dynamically feasible trajectory. An online balance compensation scheme was employed in Kuffner's work to enforce constraints on the center of gravity projection and the ZMP trajectory in order to maintain overall dynamic equilibrium.

Alternatively, works that attempt to solve for human walking motion based on performance optimization with only natural constraints imposed are believed suitable for reproduction of realistic human motions. For digital human simulations, the objective functions represent human performance measures, and optimization methods are used to solve for the feasible joint motion profiles that extremise the objective functions and satisfy the necessary constraints (Chevallereau and Aoustin 2001; Saidouni and Bessonet 2003). The importance of the works in this category is that the human motions are not artificially constrained and are dynamically feasible, and hence can realistically reproduce human motions. Optimization-based digital human motion planning problems can be viewed as numerical optimal control problems (Wang et al. 2005).

\footnotetext{
- The zero moment point (ZMP) is discussed in Section 2.1.1.
} 
While this approach is more computationally intensive and not necessarily amenable to the real-time analysis and control needed in robotics, it does yield more realistic motion and hence is better for studying the biomechanics of human locomotion.

\subsubsection{Forward vs. inverse dynamics}

Both forward and inverse dynamics formulations can be used in modeling bipedal locomotion. Forward dynamics starts with initial conditions and known forces and solves for the unknown joint displacements by numerical integration (Chevallereau et al. 1998; Roussel et al. 2001; Anderson and Pandy 2001). The process of integrating forces over time intervals to obtain walking motions can itself be computationally intensive. When one further considers that the forces that drive the bipedal locomotion are themselves unknown and must be obtained by repeatedly solving the forward dynamics problem with trial forces until those that maximize the desired human performance objectives are found, it becomes clear that approaches utilizing forward dynamics can be very computationally intensive. One way to deal with the computational intensity of forward dynamics formulations has been to use massively parallel algorithms and processing techniques (Pandy et al. 1992).

Inverse dynamics differs from forward dynamics in that it calculates unknown forces from joint displacement histories. Since the joint displacement histories associated with locomotion are unknown a priori they are determined using optimization methods (Wang et al. 2001; Bessonnet et al. 2002; Chevallereau and Aoustin 2001). The balance of joint forces that arise from joint displacements is evaluated through differential algebraic equations (DAE) and balance constraints are imposed on the optimization problem. Two important issues that arise in such inverse dynamics frameworks are the human performance criteria and methods for approximating the joint trajectories. The work of Lo et al. (2002), although it deals with human motion and tasks other than locomotion, provides a thorough description of an inverse dynamics framework for predicting human motions. By using as design variables the control points of cubic B-spline approximations of joint angle profiles, Lo et al. (2002) used a quasiNewton algorithm to solve for joint angle profiles that minimize actuating joint torques during lifting. Chevallereau and Aoustin (2001) planned a robotic walking and running motion using optimization to determine the coefficients of a polynomial approximation for profiles of the pelvis translations and joint angle rotations. Walking was treated as a combination of successive single support phases with instantaneous double support phases defined by passive impact. Saidouni and Bessonet (2003) solved for cyclic, symmetric gait motion of a 9-DOF model that moves in the sagittal plane. A cyclic, symmetric gait motion was composed of repeated gait stages with single and double support phases that are symmetric when the left and right feet change roles. The control points for the B-spline curves along with the time durations for the gait stages were optimized to minimize the actuating torque energy. By adopting the time durations as design variables the motions for both the single support and double support were simultaneously optimized.

\subsubsection{Musculoskeletal model vs. skeletal model}


Two types of models are generally used in modeling human locomotion: skeleton models in which all effects of muscles are modeled as simply as torques applied to joints; and (2) musculoskeletal models where muscle groups are included in the dynamic system and represented by Hill-type elements (Yamaguchi and Zajac 1990; Pandy et al. 1992; Pandy 2001). Skeletal models are used quite naturally in robotic modeling, and are even used in human locomotion modeling due to their relative simplicity and computational efficiency (Chevallereau and Aoustin 2001; Bessonnet et al. 2002; Saidouni and Bessonet 2003). Examples where musculoskeletal models have been employed include a 3D 8-DOF model by Yamaguchi and Zajac (1990) to restore unassisted natural gait to paraplegics and a 3D musculoskeletal body model (23 DOF, 54 muscles) for normal symmetric walking on level ground by Anderson and Pandy (2001). The forward dynamics optimization problem with such musculoskeletal models is typically posed to minimize metabolic energy expenditure per unit distance traveled. A set of terminal posture constraints is often imposed to ensure repeatability of the gait cycle, and the resulting simulations match well with the patterns of body-segmental displacements, ground-reaction forces, and muscle activations obtained from experiments.

\subsection{CURRENT WORK}

The hypothesis underlying this work is that modeling of human locomotion is best done within a combined inverse dynamics and optimization framework. A number of preceding works that have used such an approach have been focused on 2D sagittal plane walking (Bessonnet et al. 2002; Saidouni and Bessonet 2003). Here the goal is to develop a general three-dimensional formulation for modeling realistic human locomotion. Within this work foot stepping patterns are prescribed in order to overcome numerical difficulties associated with discontinuity of constraint functions that would occur otherwise. Nevertheless, gait stage durations are calculated automatically within this work by optimizing a sequence of knot variables. Like some of the previously cited works and also that of Tang et al (2003) a modified cubic B-spline time interpolation scheme is employed for the joint displacement profiles. This provides $\mathrm{C}_{2}$ continuity of the joint profiles in time and thus allows for reduction of foot impact forces. In addition, an efficient sequential quadratic programming (SQP) algorithm and software are used to solve the motion optimization problem. If suitable objective and constraint functions can be identified and utilized within this modeling framework to produce walking motions fully consistent with normal human locomotion, then a useful formulation will be obtained with which to study the biomechanics of walking. Some of the specific challenges addressed in the body of this paper are briefly described below.

\subsubsection{Treatment of multiple gait stages and discontinuity in stage transitions}

When applying gradient-based optimization to general human locomotion modeling, one has to carefully formulate the modeling problem so that the functionals used are smoothly differentiable in design space to the extent possible. If the functional gradients in design variable space are discontinuous it can result in failure to converge to optimal solutions. The first-order time derivatives of ground-foot non-slippage constraint functions tend to be discontinuous in time at the gate stage transition points. Many preceding works have avoided this potential difficulty by finding optimal human locomotion within single gait stage and then generating the remainder of the cyclic 
motion using symmetry in which the roles of left and right legs are changed [Anderson and Pandy, 2001; Kuffner et al, 2002; Azevevedo et al, 2002]. However, since general human locomotion is non-cyclic, non-symmetric and sometimes has special initial/final conditions such as zero-velocity/acceleration condition, the general formulation developed here seeks solutions for an entire general gait motion featuring multiple gait stages with different foot support patterns. For example, a gait cycle is composed of: 1) single support on left foot, 2) double support on left toes and right heel, 3) single support on right foot, and 4) double support on right toes and left heel. The current formulation can optimize gait motions over multiple gait stages. This is accomplished by dividing continuous gait motion into a number of stages and evaluating the functionals at specific points in time of each stage (for details, see Sec 3.3).

\subsubsection{Modified B-splines with stage duration design by controlling knots}

A modified cubic B-spline curve approximation method with non-repeated knots is used to interpolate joint angle and displacement profiles. The control points along with knots are optimized simultaneously to predict walking. The new formulation renders the shape functions in explicit polynomial form rather than in recursive form. Furthermore, by truncation of the first three curve segments, the remaining curve segments are given as a linear combination of a constant number of control points (four) and their corresponding basis functions. This facilitates the calculation of continuous analytical gradients and has the added benefit of saving both programming and computational efforts. Note that the shape of the basis functions change with time and knots. In the present framework, motion during a gait stage is represented by one or more curve segments bounded by two adjacent knots. By designing the knot distributions, the stage durations are optimized.

\subsubsection{Check of zero moment point (ZMP) relative to base of support (BoS)}

When using optimization formulations to solve for joint angles and displacements associated with human locomotion, various constraints are imposed to ensure that: (1) the joint angle limits are not exceeded; (2) there is no foot penetration of the ground; (3) there is no slippage of the foot on the ground; (4) the body obeys dynamic equilibrium conditions; and (5) the initial and final foot placements agree with those that are prescribed. The dynamic equilibrium condition requires equilibrium of all the forces on walking bipeds, and is herein imposed by constraining the instantaneous ZMP location to lie within the instantaneous boundaries of the base of support (BoS). The BoS corresponds to the footprint on the ground in the single support case, and to the convex hull of the foot contact regions in the double support case. The ZMP concept dates back thirty-five years and has been re-examined in recent literature for clarification and extension (Gowami 1999; Vukobratović and Borovac 2004; Sardin and Bessonnet 2004).

\subsection{OVERVIEW}

In Sec. 2, the concepts and approaches to imposing dynamic equilibrium by geometrically constraining the zero moment point (ZMP) to fall within the base of support are first introduced. Then, interpolated joint angle trajectories and their time 
derivatives are described using modified B-splines. The inertial and gravity forces acting on the body segments are also described using recursive multi-body kinematics. In Sec. 3, the optimization formulation for locomotion modeling is defined by identifying cost and constraint functions along with measures taken to ensure $C^{1}$ continuity of the cost and constraint functions in design space. The numerical examples explored in Section 4 of this paper involve three-step gait motions on level and inclined plane surfaces by a 25 degree-of-freedom virtual human model. Section 5 discusses the numerical results and issues that require further investigation as well as potential applications of this methodology in biomechanics and biomedicine.

\section{ANALYSIS}

\subsection{DYNAMIC EQUILIBRIUM}

One of the most profound issues in modeling human locomotion within an inverse dynamics framework in which only the joint displacements and rotations are known is ensuring that the resulting forces satisfy dynamic equilibrium. The ground contact forces acting on the feet are reactions to both the gravity forces and the inertial forces associated with the body's motion. The notion of the zero-moment-point (ZMP) is useful in checking dynamic equilibrium of the body and is based on the concept that the muscle forces act on the joints as self-balanced internal forces, opposite in direction and equal in magnitude. When the entire body is taken as a dynamic system undergoing prescribed displacements, the contributions of self-balanced joint forces vanish, and the gravity and inertial forces can be easily determined. The only significant unknown forces acting on the system are the ground reaction forces. If the ZMP lies within the body's base-of-support (BoS) region, it can be shown [e.g. Sardin and Bessonnet (2004)] that the body satisfies the dynamic equilibrium condition. Further elaborations on usage of the ZMP to ensure dynamic equilibrium of the human body during locomotion are described in this section.

\subsubsection{ZMP concept and implementation}

The forces acting on a walker can be sorted into: 1) ground contact forces acting on the feet; and 2) non-contact forces due to inertia and gravity. At a given instant the center of pressure (CoP) is the point of action for the resultant of the ground contact forces. The CoP is also defined as the instantaneous point on ground about which the instantaneous contact forces have a vanishing tipping moment where the tipping moment is the resultant of the contact moments that act within the ground plane. The torsional moment due to ground contact forces acts orthogonally to the ground plane and does not contribute to the tipping moment. The zero moment point (ZMP) is that point on the ground plane through which the resultant of the non-contact forces acting on the body passes. Accordingly, the non-contact forces acting on the human body exert no tipping moments about the ZMP. For balance of the tipping moments exerted on the body by the contact and non-contact forces, the CoP and ZMP points should coincide with each other. Furthermore, due to the unilateral nature of foot contact with a rigid nonadhesive walking surface, the contact stresses should be strictly compressive (or repulsive) rather than tensile (or attractive). Since the CoP necessarily lies within 
the base of support (BoS), and since the CoP and ZMP coincide, the ZMP must also reside within the BoS.

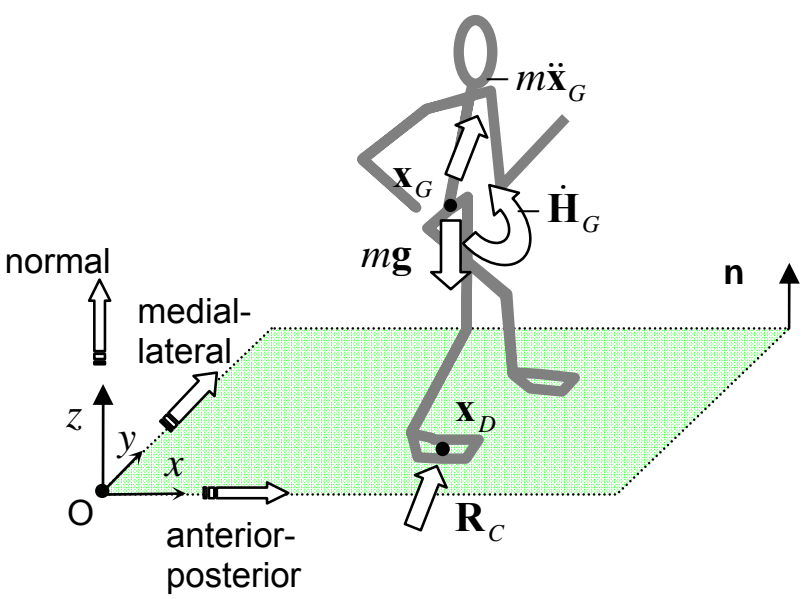

Figure 1. Forces on a walking biped including ground reaction forces $\mathbf{R}_{C}$, gravity force $m \mathbf{g}$ and inertial force $\left(-m \ddot{\mathbf{x}}_{G_{I}}\right)$ and inertial moment $\left(-\dot{\mathbf{H}}_{G}\right)$.

The resultant of non-contact forces exerted on the body (i.e. those due to inertia and gravity) is denoted by $\boldsymbol{R}^{I G F}$ and expressed as:

$$
\mathbf{R}^{I G F}=m \mathbf{g}-m \ddot{\mathbf{x}}_{G}
$$

where: $m$ is the total mass of the body; $\mathbf{g}$ the gravitational acceleration; $\mathbf{x}_{G}$ the instantaneous center of mass (CoM) of the body; and $\ddot{\mathbf{x}}_{G}$ its instantaneous acceleration. The resultant moment of $\mathbf{R}^{\mathrm{IGF}}$ about the ZMP (point $\mathbf{x}_{\mathrm{D}}$ in Figure 1 ) is given as:

$$
\mathbf{M}_{D}^{I G F}=\mathbf{x}_{D G} \times \mathbf{R}^{I G F}-\dot{\mathbf{H}}_{G}
$$

in which $\mathbf{x}_{\mathrm{DG}}=\mathbf{x}_{\mathrm{G}}-\mathbf{x}_{\mathrm{D}}$ is a vector from the ZMP point $\mathbf{x}_{\mathrm{D}}$ to the CoM $\mathbf{x}_{\mathrm{G}}$, and $\dot{\mathbf{H}}_{G}$ is the rate of angular momentum about $\mathbf{x}_{\mathrm{G}}$. If the origin of a Cartesian-coordinate system that is on the ground plane is denoted $O$ (Figure 1) the resultant moment about the point $O$, which can be written as $\mathbf{M}_{0}{ }^{\mathrm{IGF}}$ is:

$$
\mathbf{M}_{O}^{I G F}=\mathbf{x}_{G} \times \mathbf{R}^{I G F}-\dot{\mathbf{H}}_{G}
$$

Accordingly Eq. (2) can be rewritten as:

$$
\mathbf{M}_{D}^{I G F}=\mathbf{M}_{O}^{I G F}-\mathbf{x}_{D} \times \mathbf{R}^{I G F}
$$

From the condition that the tipping moment of $\boldsymbol{R}^{\prime G F}$ about the ZMP $\mathbf{x}_{D}$ vanishes one can thus write:

$$
\begin{aligned}
\mathbf{0} & =\mathbf{n} \times \mathbf{M}_{D}^{I G F} \\
& =\mathbf{n} \times \mathbf{M}_{O}^{I G F}-\left(\mathbf{n} \cdot \mathbf{R}^{I G F}\right) \mathbf{x}_{\mathrm{D}}+\left(\mathbf{n} \cdot \mathbf{x}_{D}\right) \mathbf{R}^{I G F}
\end{aligned}
$$


in which $\mathbf{n}$ is a unit vector that is normal to ground plane. Since $\mathbf{n} \cdot \mathbf{x}_{D}=0$ the position vector of the ZMP in the reference Cartesian system is obtained from Eq. (5) as follows:

$$
\mathbf{x}_{D}=\frac{\mathbf{n} \times \mathbf{M}_{O}^{I G F}}{\mathbf{n} \cdot \mathbf{R}^{I G F}}
$$

For any walking motion to satisfy dynamic equilibrium, the ZMP should stay within the base of support $\Gamma_{\text {BoS }}$.

The resulting inertia forces acting at the body's current CoM $\left(-m \ddot{\mathbf{x}}_{G}\right.$ and $\left.-\dot{\mathbf{H}}_{G}\right)$ are calculated by summing the inertia forces acting on the body's individual segments. Consequently, the resultant of the inertial and gravitational forces acting on the body $\boldsymbol{R}^{\prime G F}$ can be computed as follows:

$$
\mathbf{R}^{I G F}=\sum_{i}^{n_{\text {body }}}\left(m_{i} \mathbf{g}-m_{i} \ddot{\mathbf{x}}_{G}^{i}\right) .
$$

where: $n_{\text {body }}$ is number of body segments; $m_{i}$ denotes mass of the $i^{\text {th }}$ body segment; and $\ddot{\mathbf{x}}_{G}^{i}$ denotes the acceleration of that body segment's center of mass. In a similar fashion, the resultant moment $\boldsymbol{M}_{0}{ }^{I G F}$ of $\boldsymbol{R}^{I G F}$ about the origin of the Cartesian reference frame is computed from Eq. (3) as follows:

$$
\mathbf{M}_{O}^{I G F}=\sum_{i}^{n_{\text {body }}}\left[\mathbf{x}_{G}^{i} \times\left(m_{i} \mathbf{g}-m_{i} \ddot{\mathbf{x}}_{G}^{i}\right)-\dot{\mathbf{H}}_{G}^{i}\right]
$$

in which $\mathbf{x}_{G}^{i}$ is the position vector of the $i^{\text {th }}$ body segment's center of mass in the reference Cartesian frame and $\dot{\mathbf{H}}_{G}^{i}$ is the rate of angular momentum about $\mathbf{x}_{G}^{i}$. In the current modeling framework, the rate of angular momentum of each body segment about its own center of mass is neglected (i.e. $\dot{\mathbf{H}}_{G}^{i}=\mathbf{0}$ ) on the assumption that the mass of each body link is concentrated at its center of mass $\mathbf{x}_{G}^{i}$.

\subsubsection{Foot contact, stepping patterns, and collision avoidance}

For evaluation of dynamic equilibrium during $3 \mathrm{D}$ walking, the BoS $\left(\Gamma_{B o S}\right.$ in Figure 2$)$ should be expressed in mathematical form according to different foot stepping patterns. The stepping patterns refer to which foot-segments are in ground contact during different gait stages. Figure 3 shows that each foot has six potential contact points with the right foot having the right toe medial (RT1), right toe lateral (RT2), right ball medial (RB1), right ball lateral (RB2), right heel medial (RH1), right heel lateral $(\mathrm{RH} 2)$. The left foot has six analogous contact points. For the double support phase of the gate with right foot in the front (denoted by RDS), the rear part of right foot (RB1-RB2-RH1-RH2) and the front part of left foot (LT1-LT2-LB1-LB2) are in contact with the ground (Fig. 3). The phase of the gait that has only the right foot on the ground is denoted RSS for rightfoot-single-support. In this phase all six points of the right foot are in ground contact. Similarly, during left-foot-single-support (LSS) all six points of the left foot are in ground contact. All of the foot contact points for the four gait stages are defined in Table 1. 


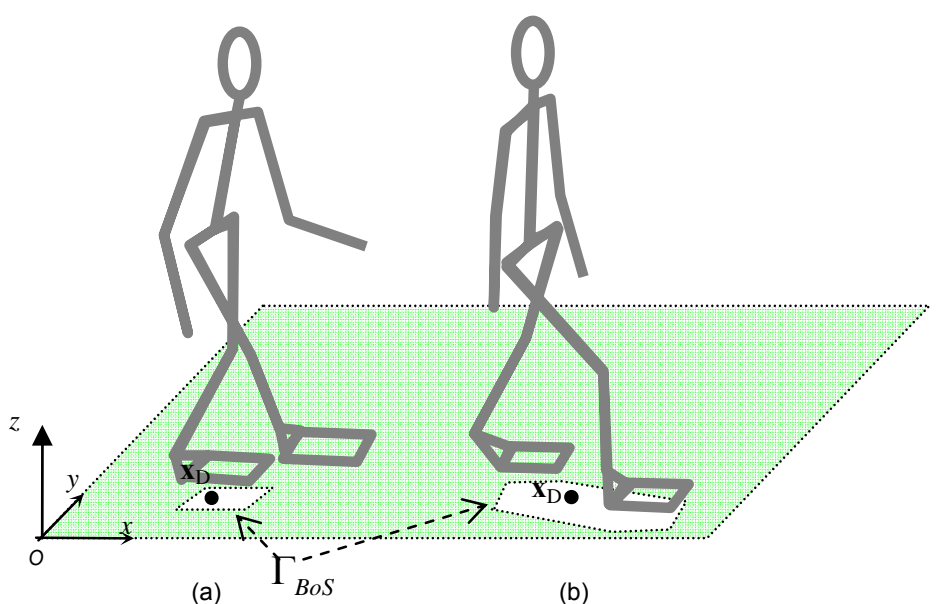

Figure 2. Illustration of base of support $\Gamma_{B o S}$ in (a) single support phase (SSP); and (b) double support phase (DSP).

Table 1: Foot-points in ground contact with different stepping patterns. Symbol ' $Y$ ' means that the footpoint is in ground contact, and symbol ' $N$ ' means that the foot-point is not.

\begin{tabular}{|l|l|l|l|l|l|l|l|l|l|l|l|l|}
\hline STEP PATTERN & LH1 & LH2 & LB1 & LB2 & LT1 & LT2 & RH1 & RH2 & RB1 & RB2 & RT1 & RT2 \\
\hline RDS & $\mathrm{N}$ & $\mathrm{N}$ & $\mathrm{Y}$ & $\mathrm{Y}$ & $\mathrm{Y}$ & $\mathrm{Y}$ & $\mathrm{Y}$ & $\mathrm{Y}$ & $\mathrm{Y}$ & $\mathrm{Y}$ & $\mathrm{N}$ & $\mathrm{N}$ \\
\hline RSS & $\mathrm{N}$ & $\mathrm{N}$ & $\mathrm{N}$ & $\mathrm{N}$ & $\mathrm{N}$ & $\mathrm{N}$ & $\mathrm{Y}$ & $\mathrm{Y}$ & $\mathrm{Y}$ & $\mathrm{Y}$ & $\mathrm{Y}$ & $\mathrm{Y}$ \\
\hline LDS & $\mathrm{Y}$ & $\mathrm{Y}$ & $\mathrm{Y}$ & $\mathrm{Y}$ & $\mathrm{N}$ & $\mathrm{N}$ & $\mathrm{N}$ & $\mathrm{N}$ & $\mathrm{Y}$ & $\mathrm{Y}$ & $\mathrm{Y}$ & $\mathrm{Y}$ \\
\hline RDS & $\mathrm{Y}$ & $\mathrm{Y}$ & $\mathrm{Y}$ & $\mathrm{Y}$ & $\mathrm{Y}$ & $\mathrm{Y}$ & $\mathrm{N}$ & $\mathrm{N}$ & $\mathrm{N}$ & $\mathrm{N}$ & $\mathrm{N}$ & $\mathrm{N}$ \\
\hline
\end{tabular}

The BoS polygon and its interior $\Gamma_{\text {Bos }}$ can be determined for each foot contact pattern. For example, during single foot support conditions (either LSS or RSS), the BoS polygon is a simple quadrilateral. Under LSS, the corners of the quadrilateral are coordinates of the four contact points LH2, LH1, LT1, LT2, and under RSS, the corners of the quadrilateral are the coordinates of RH2, RH1, RT1, RT2. Under double foot support conditions, the BoS will be formed by the intersection of six sub-regions each of which is a half-plane denoted by $L_{i}$ (Figure 3 ). In the general case, $\Gamma_{B o S}$ is formed by the intersection of $n_{B o s}$ half-planes as follows:

$$
\Gamma_{\text {BoS }}=\bigcap_{i=1}^{n_{\text {BoS }}} L_{i}
$$

Each of the half-planes can be expressed as follows:

$$
\begin{aligned}
L_{i} & =\left\{\mathbf{x} \mid b\left(\mathbf{x}, \mathbf{x}_{\eta_{i 1}}, \mathbf{x}_{\eta_{i 2}}\right) \leq 0\right\}, \\
b\left(\mathbf{x}, \mathbf{x}_{\eta_{i 1}}, \mathbf{x}_{\eta_{i 2}}\right) & =\mathbf{n} \cdot\left[\left(\mathbf{x}-\mathbf{x}_{\eta_{i 2}}\right) \times\left(\mathbf{x}_{\eta_{i 1}}-\mathbf{x}_{\eta_{i 2}}\right)\right]
\end{aligned}
$$

In the equation above, $b\left(\mathbf{x}, \mathbf{x}_{\eta_{11}}, \mathbf{x}_{\eta_{12}}\right)$ is the distance that a point $\mathbf{x}$ lies above a line connecting the location of the two foot-points $\mathbf{x}_{\eta_{i 1}}$ and $\mathbf{x}_{\eta_{i 2}}$. Appropriate pairs of foot- 
points $\left\{\eta_{i 1}, \eta_{i 2} \mid 1 \leq i \leq n_{B o S}\right\}$ that define the boundary of $\Gamma_{B o S}$ for different foot support conditions are provided in Table 2.

The same basic ideas can be used to create foot collision avoidance (FCA) constraint that prevents footprints of the left and right feet from overlapping each other. In the current framework this is accomplished by not allowing the right foot to lie within the left foot's lateral half-plane, and not allowing the left foot to fall within the lateral plane of the right foot. Mathematically, the foot collision avoidance condition is represented as:

$$
\begin{aligned}
& \mathbf{x}_{R H 1}, \mathbf{x}_{R T 1} \in\left(L_{m 1}\right)^{C}=\left\{\mathbf{x} \mid b\left(\mathbf{x}, \mathbf{x}_{\mathrm{LH} 1}, \mathbf{x}_{\mathrm{LT} 1}\right) \leq 0\right\} \\
& \mathbf{x}_{L H 1}, \mathbf{x}_{L T 1} \in\left(L_{\mathrm{m} 2}\right)^{C}=\left\{\mathbf{x} \mid b\left(\mathbf{x}, \mathbf{x}_{\mathrm{RT} 1}, \mathbf{x}_{\mathrm{RH} 1}\right) \leq 0\right\}
\end{aligned}
$$

where $b\left(\mathbf{x}, \mathbf{x}_{\eta_{m 1}}, \mathbf{x}_{\eta_{m 2}}\right)$ is the distance of a point $\mathbf{x}$ above a line connecting two foot-points $\mathbf{x}_{\eta_{m 1}}$ and $\mathbf{x}_{\eta_{m 2}}$.

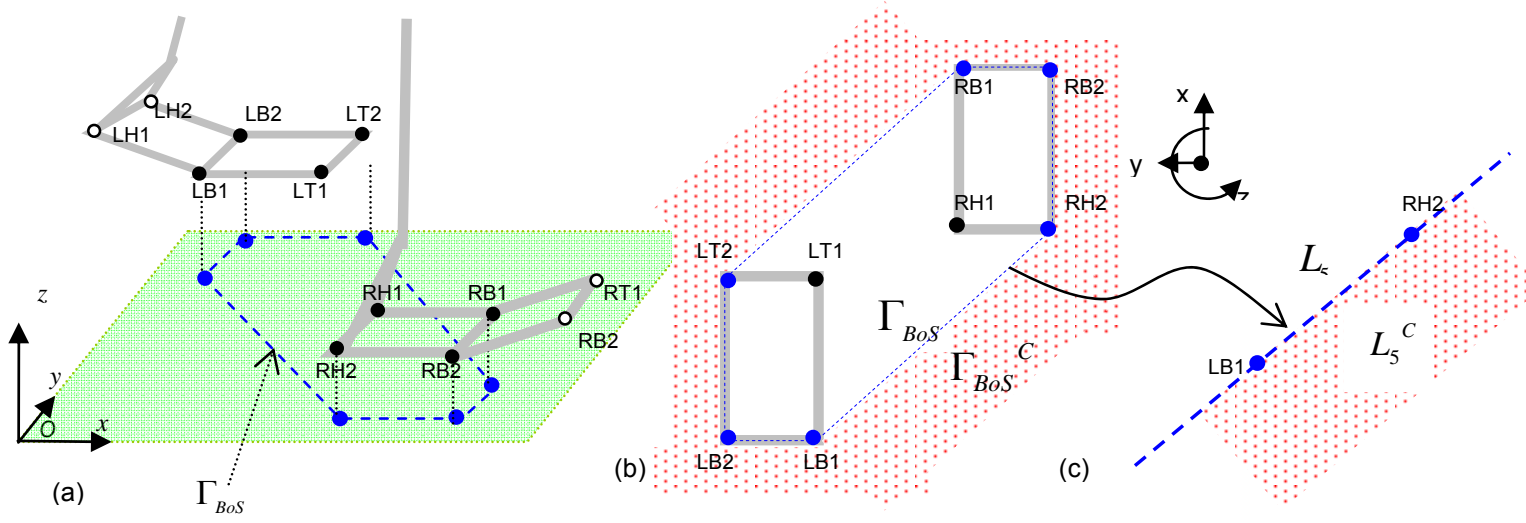

Figure 3. The BoS polygon during right-foot-double-support (RDS): (a) side view and (b) plan view. $(\mathrm{LH} 1 / \mathrm{LH} 2=$ medial/lateral left foot heel, $\mathrm{LB} 1 / \mathrm{LB} 2=$ medial/lateral left foot-ball, $\mathrm{LT} 1 / \mathrm{LT} 2=$ medial/lateral left foot toe, $\mathrm{RH} 1 / \mathrm{RH} 2=$ medial/lateral right foot heel, $\mathrm{RB} 1 / \mathrm{RB} 2=$ medial/lateral right foot-ball, $\mathrm{RT} 1 / \mathrm{RT} 2=$ medial/lateral right foot toe). The BoS region $\Gamma_{\mathrm{BoS}}$ is defined in Eq. (9) and is an intersection of up to six half-planes $L_{i}$ defined in Eq. (10) and $\Gamma_{B O S}^{C}$ denotes its complement with $L_{5}^{C}$ the complement of $L_{5}$ as shown in (c).

Table 2. Foot contact points defining boundaries of the base-of-support BoS for various foot support conditions. The parameter $n_{B o S}$ denotes the number of $L_{j}$ segments defining the

\begin{tabular}{|c|c|c|c|c|c|c|c|c|c|c|c|c|c|}
\hline \multirow{2}{*}{$\begin{array}{l}\text { STEP } \\
\text { PATTERN }\end{array}$} & \multirow{2}{*}{$n_{B o S}$} & \multicolumn{2}{|l|}{$L_{1}$} & \multicolumn{2}{|l|}{$L_{2}$} & \multicolumn{2}{|l|}{$L_{3}$} & \multicolumn{2}{|l|}{$L_{4}$} & \multicolumn{2}{|l|}{$L_{5}$} & \multicolumn{2}{|l|}{$L_{6}$} \\
\hline & & $\eta_{11}$ & $\eta_{12}$ & $\eta_{21}$ & $\eta_{22}$ & $\eta_{31}$ & $\eta_{32}$ & $\eta_{41}$ & $\eta_{42}$ & $\eta_{51}$ & $\eta_{52}$ & $\eta_{61}$ & $\eta_{62}$ \\
\hline LSS & 4 & LH2 & LT2 & LT2 & LT1 & LT1 & LH1 & LH1 & LH2 & - & - & - & - \\
\hline RSS & 4 & $\mathrm{RH} 1$ & RT1 & RT1 & RT2 & RT2 & $\mathrm{RH} 2$ & $\mathrm{RH} 2$ & $\mathrm{RH} 1$ & - & - & - & - \\
\hline
\end{tabular}
boundary, with each segment defined by two foot contact points $\eta_{j 1}$ and $\eta_{j 2}$ 


\begin{tabular}{|l|l|l|l|l|l|l|l|l|l|l|l|l|l|}
\hline LDS & 6 & LH2 & LB1 & LB1 & RT1 & RT1 & RT2 & RT2 & RB2 & RB2 & RB1 & RB1 & LH2 \\
\hline RDS & 6 & LB2 & LT2 & LT2 & RB1 & RB1 & RB2 & RB2 & RH2 & RH2 & LB1 & LB1 & LB2 \\
\hline
\end{tabular}

\subsection{TIME INTERPOLATION OF JOINT DEGREES OF FREEDOM WITH MODIFIED CUBIC B-SPLINES}

The joint degrees of freedom of the human model are approximated in time using linear combinations of modified cubic B-spline basis functions. This approximation method basically corresponds to the unclamped cubic B-spline approximation (Piegl andTiller, 1997) but differs only in that it excludes irregular segments. An irregular segment has insufficient number of control parameters. For example, a linear combination of less than four cubic basis functions cannot represent an arbitrary cubic curve. The modified cubic B-spline turns out to be a cubic uniform B-spline curve when knots are uniformly spaced.

The global time interval on which a motion planning problem is solved is denoted by $\Omega=\left\{t_{\min }, t_{\max }\right\}$. With simple knot approximations, a sequence of monotonically increasing time values on the global interval are called knots and the number of values in the sequence is denoted nknots. The set of knots is represented as follows: $\left\{t_{0}, t_{1}, t_{2}, \ldots, t_{n k n o t s-1}\right\}$ with $t_{0}=t_{\min }$ and $t_{\text {nknots }-1}=t_{\max }$. The global time interval is partitioned into a discrete set of segments defined by these knots as follows:

$$
\Omega=\bigcup_{i=0}^{i=n k n o t s-2} \Omega_{i} \text { where }\left\{\Omega_{i}=t \mid t_{i} \leq t<t_{i+1}\right\}
$$

The modified cubic B-spline curve approximates a model degree-of-freedom $q$ as follows:

$$
q\left(t \in \Omega_{i}\right)=\sum_{j=0}^{j=3} \hat{q}_{(i+j-3)} M_{i j}\left(t, \mathbf{t}^{i}\right) \quad \mathrm{i} \in\{3,4, \ldots,(\text { nknots }-3)\}
$$

where: $\hat{q}_{k}$ denotes the control points, $M_{i j}$ denotes the basis function that covers $i^{\text {th }}$ segment, i.e. $t \in \Omega_{i}$, and $\mathbf{t}^{i}$ denotes the set of bounding knot values of the segments that the basis functions $M_{i j}$ span, specifically, $\mathbf{t}^{i}=\left\{t_{i-3}, t_{i-2}, t_{i-1}, t_{i}, t_{i+1}, t_{i+2}\right\}$. Note that, for the curve segment (i=1,2,3 or nknots-2), some knots necessary for constructing $M_{i j}$ are missing, and it makes the curve segment irregular.

The number of regular segments (nseg) corresponding to the global time interval with (nkots) knots is: nseg $=n k n o t s-5$. The number of control points needed for the approximation is: $n c t r l=n s e g+3=n k n o t s-2$. The shape functions that cover the $i^{\text {th }}$ segment $\Omega_{i}$ are as follows:

$$
M_{i 0}\left(t, \mathbf{t}^{i}\right)=\frac{\left(t_{i+1}-t\right)^{3}}{\left(t_{i+1}-t_{i-2}\right)\left(t_{i+1}-t_{i-1}\right)\left(t_{i+1}-t_{i}\right)}
$$




$$
\begin{aligned}
& M_{i 1}\left(t, \mathbf{t}_{i}\right)=\frac{\left(t-t_{i+1}\right)^{2}\left(t-t_{i-2}\right)}{\left(t_{i+1}-t_{i-2}\right)\left(t_{i+1}-t_{i-1}\right)\left(t_{i+1}-t_{i}\right)}+\frac{\left(t-t_{i-1}\right)\left(t-t_{i+1}\right)\left(t-t_{i+2}\right)}{\left(t_{i+1}-t_{i-1}\right)\left(t_{i+1}-t_{i}\right)\left(t_{i+2}-t_{i-1}\right)}+\frac{\left(t-t_{i+2}\right)^{2}\left(t-t_{i}\right)}{\left(t_{i+1}-t_{i}\right)\left(t_{i+2}-t_{i-1}\right)\left(t_{i+2}-t_{i}\right)} \\
& M_{i 2}\left(t, \mathbf{t}_{i}\right)=\frac{\left(t_{i-1}-t\right)^{2}\left(t_{i+1}-t\right)}{\left(t_{i+1}-t_{i-1}\right)\left(t_{i+1}-t_{i}\right)\left(t_{i+2}-t_{i-1}\right)}+\frac{\left(t_{i-1}-t\right)\left(t_{i}-t\right)\left(t_{i+2}-t\right)}{\left(t_{i+1}-t_{i}\right)\left(t_{i+2}-t_{i-1}\right)\left(t_{i+2}-t_{i}\right)}+\frac{\left(t_{i}-t\right)^{2}\left(t_{i+3}-t\right)}{\left(t_{i+1}-t_{i}\right)\left(t_{i+2}-t_{i}\right)\left(t_{i+3}-t_{i}\right)} \\
& M_{i 3}\left(t, \mathbf{t}_{i}\right)=\frac{\left(t-t_{i}\right)^{3}}{\left(t_{i+1}-t_{i}\right)\left(t_{i+2}-t_{i}\right)\left(t_{i+3}-t_{i}\right)}
\end{aligned}
$$

\subsection{TIME SEGMENT DURATIONS}

The sequence of monotonically increasing knots noted above is not chosen arbitrarily but instead obtained by optimizing the duration of time segments. A set of additional optimization variables $\hat{\mathbf{d}}=\left\{\hat{d}_{j} \mid 0 \leq j<n_{\text {seg }}+2 ; 0<d^{\text {min }} \leq \hat{d}_{j}\right\}$ is thus introduced to represent the individual time segment durations where $\hat{d}_{i}$ denotes a stage duration and, $d^{\text {min }}$ is the minimum permissible value allowed (in this study $d^{\text {min }}=0.1$ seconds is used). The knot variables are in turn expressed from the segment durations as follows:

$$
t_{i}=t_{\min }^{\operatorname{sim}}+\sum_{j=0}^{i-1} \hat{d}_{j}-\left(\hat{d}_{0}+\hat{d}_{1}\right), \quad \text { and }(0 \leq i<n s e g+3)
$$

By Eq. (15), the monotonicity of the knot sequence is assured since the segment durations are all positive. Furthermore, the value of the third knot $t_{2}$ which begins the first regular time segment becomes the start time of simulation. $t_{\min }^{\text {sim }}$. When the problem end time $t_{\max }^{\text {sim }}$ is specified, an additional constraint on the final time is imposed such that:

$$
t_{\text {nseg }+3}=t_{\min }^{\text {sim }}+\sum_{j=3}^{n s e g+2} \hat{d}_{j}=t_{\max }^{\text {sim }} .
$$

The derivatives of time-interpolated joint degrees of freedom, and their velocities and accelerations can be obtained quite straightforwardly with respect to both the control variables and also the segment duration variables.

\subsection{KINEMATICS}

The Denavit-Hartenberg method [Denavit and Hartenberg (1955); Featherstone (1987)] is used in the present human model to represent the relative motions of a $3 \mathrm{D}$ rigid skeletal linkage system. As shown in Fig. 4, the human model has 28 rotational degrees of freedom and three rigid body translational degrees of freedom that govern the location of the pelvis.

A neutral state of the skeletal model is one in which all of the joint rotation angles assume values of zero. A local coordinate system is attached to each skeletal body segment between the joints shown in Fig. 4. The relationship between the coordinates 
of a point $P$ on the $i^{\text {th }}$ segment and the coordinates of a point $Q$ on the $n^{\text {th }}$ segment are given by a relationship of the following form:

$$
\tilde{\mathbf{x}}_{Q}=\left(\prod_{j=i}^{n-1} \mathbf{T}_{j}^{j+1}\left(q_{j}\right)\right) \cdot \tilde{\mathbf{x}}_{P}
$$

where $\tilde{\mathbf{x}}_{P} \equiv\left\{\mathbf{x}_{P}, 1\right\}$ is an augmented $4 \mathrm{X} 1$ vector for $\mathbf{x}_{P} ; \tilde{\mathbf{x}}_{Q} \equiv\left\{\mathbf{x}_{Q}, 1\right\} ; q_{j}$ is a relative rotation angle of the $j^{\text {th }}$ local coordinate system with respect to the $(j-1)^{\text {th }}$ coordinate system; and $\mathbf{T}^{j}\left(q_{j}\right)$ is a $4 \times 4$ transformation matrix from $(j-1)^{\text {th }}$ to the $j^{\text {th }}$ coordinate system. Let the transformation matrix from $N^{\text {th }}$ local to global Cartesian coordinates be denoted as $\mathbf{S}^{N}$ :

$$
\mathbf{S}^{N}=\prod_{i=1}^{N} \mathbf{T}^{i}\left(q_{i}\right) .
$$

By the recursive calculation of kinematical quantities [Featherstone (1987)], the computation procedure can be simplified as follows. The transformation matrix $\mathbf{S}^{N}$ is:

$$
\mathbf{S}^{N}=\left[\begin{array}{ll}
\mathbf{S}^{N-1} \cdot \mathbf{T}^{N}\left(q_{N}\right) & (N>0) \\
\mathbf{I} & (N=0)
\end{array}\right.
$$

where $\mathbf{I}$ is a $4 \mathrm{X} 4$ identity matrix.

The velocity of point $\mathrm{P} \dot{\mathbf{x}}_{P}^{0}$ is calculated from the relation:

$$
\dot{\tilde{\mathbf{x}}}_{P}=\frac{d}{d t}\left[\mathbf{S}^{N} \cdot \widetilde{\mathbf{x}}_{P}^{N}\right]=\dot{\mathbf{S}}^{N} \cdot \tilde{\mathbf{x}}_{P}^{N}
$$

where $\dot{\widetilde{\mathbf{x}}}_{P} \equiv\left\{\dot{\mathbf{x}}_{P}, 1\right\}$ is a $4 \mathrm{X} 1$ vector for $\dot{\mathbf{x}}_{P}$, and a velocity transformation matrix $\dot{\mathbf{S}}^{N}$ is the first time-derivative of $\mathbf{S}^{N}$. The velocity transformation matrix is given in recursive form as:

$$
\dot{\mathbf{S}}^{N}=\left[\begin{array}{ll}
\dot{\mathbf{S}}^{N-1} \cdot \mathbf{T}^{N}\left(q_{N}\right)+\mathbf{S}^{N-1} \cdot \frac{\partial \mathbf{T}^{N}\left(q_{N}\right)}{\partial q_{N}} \dot{q}_{N} & (N>0) \\
\mathbf{0} & (N=0)
\end{array}\right.
$$

The acceleration of point $P, \ddot{\mathbf{x}}_{P}$ is expressed as:

$$
\ddot{\tilde{\mathbf{x}}}_{P}=\frac{d^{2}}{d t^{2}}\left[\mathbf{S}^{N}(\mathbf{Q}(t)) \cdot \widetilde{\mathbf{x}}_{P}^{N}\right]=\ddot{\mathbf{S}}^{N} \cdot \widetilde{\mathbf{x}}_{P}^{N}
$$

where $\ddot{\widetilde{\mathbf{x}}}_{P} \equiv\left\{\ddot{\mathbf{x}}_{P}, 1\right\}$ is a $4 \mathrm{X} 1$ vector for $\ddot{\mathbf{x}}_{P}$, and $\ddot{\mathbf{S}}^{N}$ is an acceleration transformation matrix that is the second time-derivative of $\mathbf{S}^{N}$ given in recursive form as: 


$$
\ddot{\mathbf{S}}^{N}=\left[\begin{array}{ll}
\ddot{\mathbf{S}}^{N-1} \cdot \mathbf{T}^{N}\left(q_{N}\right)+2 \dot{\mathbf{S}}^{N-1} \cdot \frac{\partial \mathbf{T}^{N}\left(q_{N}\right)}{\partial q_{N}} \dot{q}_{N}+\mathbf{S}^{N-1} \cdot\left(\frac{\partial^{2} \mathbf{T}^{N}\left(q_{N}\right)}{\partial q_{N}{ }^{2}} \dot{q}_{N}{ }^{2}+\frac{\partial \mathbf{T}^{N}\left(q_{N}\right)}{\partial q_{N}} \ddot{q}_{N}\right) & (N>0) \\
\mathbf{0} & (N=0)
\end{array}\right.
$$

The gradient of transformation matrices $\mathbf{S}^{N}, \dot{\mathbf{S}}^{N}$ and $\ddot{\mathbf{S}}^{N}$ with respect to the kinematic quantities of $q_{k}, \dot{q}_{k}$ and $\ddot{q}_{k}$ can be evaluated in a very straightforward manner.

\section{VIRTUAL HUMAN MODEL LOCOMOTION FORMULATION}

\subsection{PROBLEM STATEMENT}

The optimization problem to be solved for dynamic motion planning of human gait is expressed as follows for the 31-DOF skeletal model of Fig. 4:

Find:

$$
\boldsymbol{\Theta}=\left\{\hat{q}_{k i}, \hat{d}_{j}\right\} \quad\left(0 \leq i<n_{\text {crrl }} ; \quad 0 \leq k<n_{\text {dof }} ; \quad 0 \leq j<n_{\text {crrl }}-1\right)
$$

that minimize:

$$
\begin{aligned}
F(\boldsymbol{\Theta}) & =\int_{0}^{T} f^{\cos t}(\mathbf{X}(\boldsymbol{\Theta}, t)) d t \\
& \cong \sum_{j}\left\{\sum_{p}^{n_{\text {samp }}} f^{\cos t}\left(\mathbf{X}\left(\boldsymbol{\Theta}, s_{p}^{j}\right)\right)\left(\frac{\hat{d}_{j}}{n_{\text {samp }}}\right)\right\}
\end{aligned}
$$

subject to:

$$
\begin{array}{rlrl}
H_{l}(\boldsymbol{\Theta}) & =\sum_{j} \sum_{p}^{n_{\text {seg }} n_{\text {sanp }}}\left[h_{l}^{+}\left(\mathbf{X}\left(\boldsymbol{\Theta}, s_{j p}\right)\right)\right]^{2} & & \left(0 \leq l<n_{\text {cnstr }}\right) \\
& =0 & & \text { (equality) } \\
h_{l}^{+} & =\left[\begin{array}{ll}
h_{l}(\mathbf{X}(\boldsymbol{\Theta}, t)) & \text { inequality) }
\end{array}\right.
\end{array}
$$

where $\Theta$ is the set of design variables that consists of the control points $\hat{q}_{k i}$ and stage durations $\hat{d}_{j} ; F(\boldsymbol{\Theta})$ is a cost function that is the integral of the instantaneous performance measure $f^{\text {cost }}$ over time, and is approximated by a finite sum of function values at sample time points; $H_{l}(\boldsymbol{\Theta})$ is a square sum of the violation $h_{l}^{+}$of the imposed conditions that are sorted into equality constraints $h_{l}(\Theta, t)=0$ and inequality constraints $g_{l}(\Theta, t) \leq 0 ; s_{j p}$ is the sample time frame, and is another function of design variables, as will be explained in the Sec. 3.3.2. 
Although the optimization problem posed above is extremely general, specificity is gained by selecting specific objective and constraint functions. In the following subsections a number of functions that can be used as either the objective or constraints are presented. Then in Section 4, the formulation is exercised with a specific objective and set of constraints. The optimization problem is solved using gradient-based sequential quadratic programming methods. It is noted here that to solve the optimization problem efficiently, all the equality and inequality constraints are collapsed into one equality constraint in Eq. (26.b).

Dimensions $(\mathrm{cm})$
\begin{tabular}{|l|r|}
\hline $\mathrm{L}_{0}$ & 5.63 \\
\hline $\mathrm{L}_{1}$ & 5.44 \\
\hline $\mathrm{L}_{2}$ & 6.00 \\
\hline $\mathrm{L}_{3}$ & 18.13 \\
\hline $\mathrm{L}_{4}$ & 12.57 \\
\hline $\mathrm{L}_{5}$ & 30.48 \\
\hline $\mathrm{L}_{6}$ & 24.74 \\
\hline $\mathrm{L}_{7}$ & 8.52 \\
\hline $\mathrm{L}_{8}$ & 9.00 \\
\hline $\mathrm{L}_{9}$ & 38.26 \\
\hline $\mathrm{L}_{10}$ & 39.45 \\
\hline $\mathrm{L}_{11}$ & 9.00 \\
\hline $\mathrm{L}_{12}$ & 9.00 \\
\hline $\mathrm{L}_{13}$ & 10.00 \\
\hline
\end{tabular}

Mass distribution $(\mathrm{kg})$

\begin{tabular}{|l|r|}
\hline Trunk & 35.00 \\
\hline Pelvis & 14.00 \\
\hline $\begin{array}{l}\text { Upper } \\
\text { arm }\end{array}$ & 2.24 \\
\hline $\begin{array}{l}\text { Forearm, } \\
\text { hand }\end{array}$ & 1.78 \\
\hline Thigh & 8.00 \\
\hline Shank & 3.72 \\
\hline $\begin{array}{l}\text { Hind foot, } \\
\text { toes }\end{array}$ & 1.16 \\
\hline Total & 82.8 \\
\hline
\end{tabular}

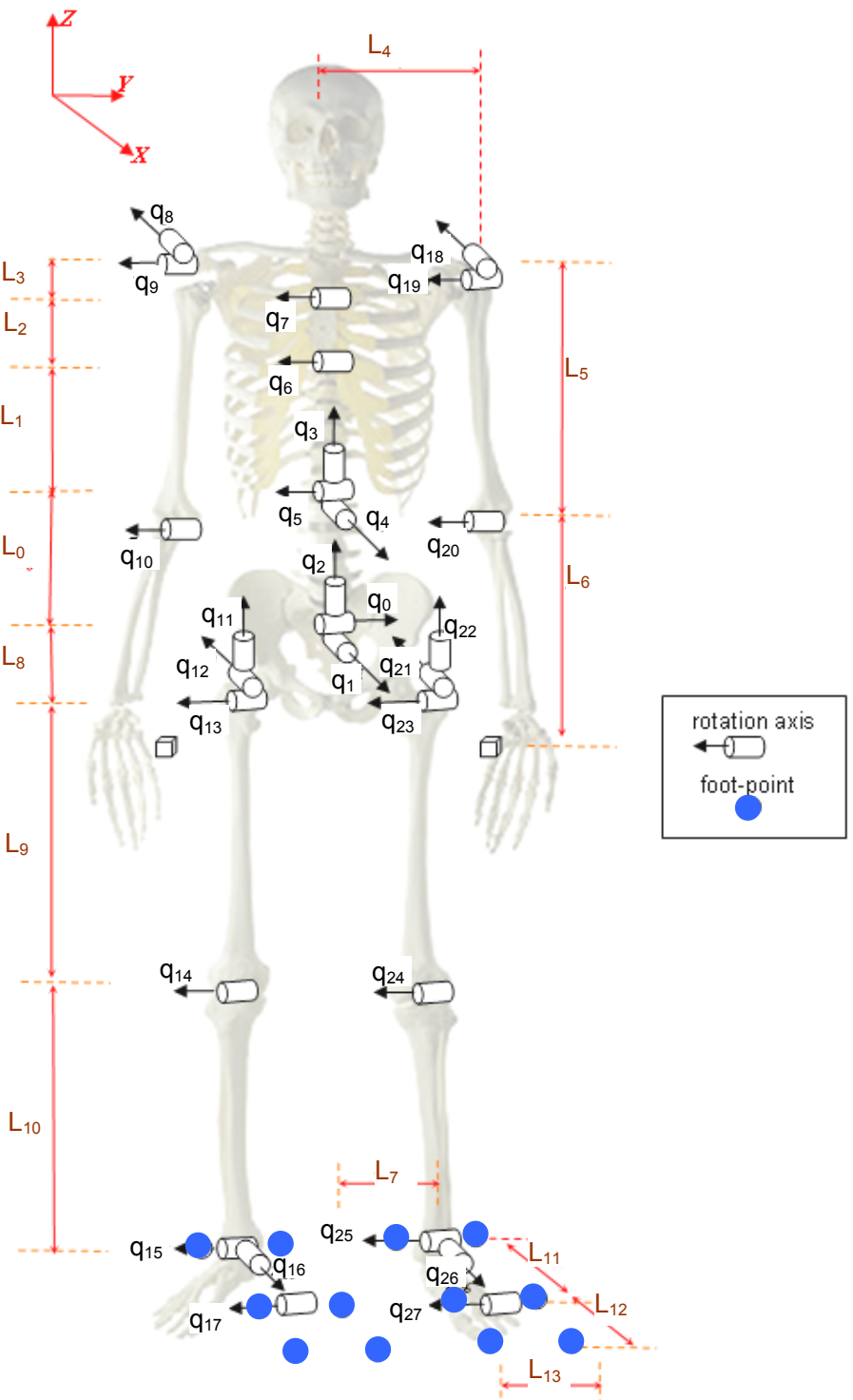

Figure 4. The 31-DOF human linkage model with dimensions $\left(L_{0}-L_{13}\right)$ and joint rotation axes $\left(q_{0}-q_{27}\right)$ denoted by cylinders. Three additional degrees of freedom $\left(q_{28}-q_{30}\right)$ capture rigid body translation of the body. Specific segment lengths and mass values represent the values used in the computations of Section 4. 


\subsection{SPECIFIC COST AND CONSTRAINT FUNCTIONS}

\subsubsection{Upright trunk posture (UTP)}

A potential objective or constraint function involves deviation of the trunk from an upright trunk posture (UTP) which is quantified with the following function

$$
f^{u t p}(\boldsymbol{\Theta}, t)=\left\|\left(\mathbf{x}_{p e l v}-\mathbf{x}_{s p 3}\right) \times \mathbf{g}\right\|^{2}
$$

where $\mathbf{x}_{p e l v}$ and $\mathbf{x}_{s p 3}$ are respectively the Cartesian coordinates of the pelvic joint center associated with $\boldsymbol{q}_{0}$ and the third spinal joint joint center associated with $\boldsymbol{q}_{7}$ ), and $\mathbf{g}$ is the gravity vector.

\subsubsection{Dynamic equilibrium (DE)}

The dynamic equilibrium condition is to enforce equilibrium of all gravitational and inertial forces acting on the body. This is assured in the current framework by constraining the ZMP whose coordinate is denoted $\mathbf{x}_{D}$ and is given by Eq. (6) to lie within $\Gamma_{\text {BoS }}$. The corresponding constraint is specified for $\mathbf{x}_{D}$ relative to each of the bounding segments of $\Gamma_{B O S}$ as follows.

$$
g_{i}^{d e}(\boldsymbol{\Theta}, t)=b\left[\mathbf{x}_{D}(\boldsymbol{\Theta}, t), \mathbf{x}_{\eta_{i 1}}\left(\Psi_{C}(t)\right), \mathbf{x}_{\eta_{i 2}}\left(\Psi_{C}(t)\right)\right] \leq 0, \quad\left(1 \leq i \leq n_{\text {Bos }}\right)
$$

The vertices of $\Gamma_{\text {Bо }}$ for varying foot support conditions were specified in Table 2 .

\subsubsection{Foot collision avoidance (FCA)}

Foot collision avoidance in the model keeps the left and right feet from occupying the same ground points simultaneously during double support conditions and is imposed herein with the following four individual constraints whose symbols and details are further explained in Eqs. (10) and (11), Fig. 5, and Table 3.

$$
\begin{aligned}
& g_{1}^{f c a}=b\left[\mathbf{x}_{L H 1}, \mathbf{x}_{R H 1}, \mathbf{x}_{R T 1}\right] \leq 0 \\
& g_{2}^{f c a}=b\left[\mathbf{x}_{L T 1}, \mathbf{x}_{R B 1}, \mathbf{x}_{R T 1}\right] \leq 0 \\
& g_{3}^{f c a}=b\left[\mathbf{x}_{R H 1}, \mathbf{x}_{L H 1}, \mathbf{x}_{L T 1}\right] \leq 0 \\
& g_{4}^{f c a}=b\left[\mathbf{x}_{R T 1}, \mathbf{x}_{L H 1}, \mathbf{x}_{L T 1}\right] \leq 0
\end{aligned}
$$

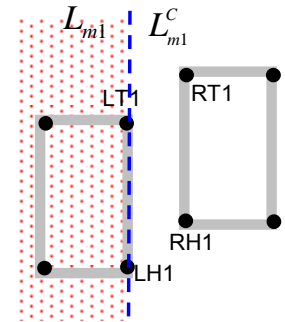

(a)

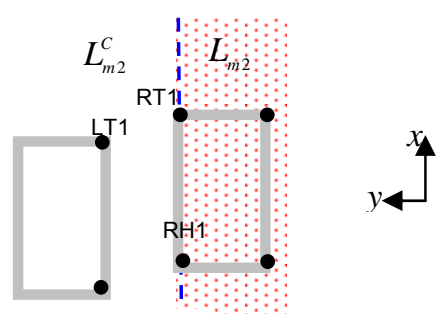

(b)

Figure 5. Aids to foot collision avoidance during RDS: (a) at left the half-plane $L_{m 1}$ lateral to the medial edge of the left foot and $\left(L_{m 1}\right)^{\mathrm{C}}$ its complement; and (b) at right the half-plane $L_{m 2}$ lateral to the medial edge of the right foot and $\left(L_{m 2}\right)^{C}$ its complement. 


\subsubsection{Joint angle limit (JAL)}

The limits on the rotation or translation of each joint are enforced by imposing upper and lower bounds as follows:

$$
\begin{aligned}
& g_{k}^{\text {jal }}(\boldsymbol{\Theta}, t)=q_{k}^{\min }-q_{k}(\boldsymbol{\Theta}, t) \leq 0 \\
& g_{k+n_{\text {dof }}}^{\text {jal }}(\boldsymbol{\Theta}, t)=q_{k}(\boldsymbol{\Theta}, t)-q_{k}^{\max } \leq 0
\end{aligned} \quad\left(1 \leq k \leq n_{\text {dof }} \quad t \in\left[t_{\text {min }}, t_{\max }\right]\right)
$$

where $q_{k}$ is the $k^{\text {th }}$ degree of freedom representing either joint angle or pelvis translation at time frame $t$ and, $q_{k}^{\min }$ and $q_{k}^{\max }$ are its upper and lower limits respectively.

\subsubsection{Initial / final foot locations (IFL)}

To provide starting and ending locations for the modeled walking process, the locations of the left toe are specified at the problem start and end times, respectively with two equality constraints expressed as follows:

$$
\begin{aligned}
& h_{1}^{i f l}(\boldsymbol{\Theta})=\mathbf{x}_{L T 1}\left(\boldsymbol{\Theta}, t_{\min }\right)-\mathbf{x}^{\text {init }}=0 \\
& h_{2}^{\text {ifl }}(\boldsymbol{\Theta})=\mathbf{x}_{L T 1}\left(\boldsymbol{\Theta}, t_{\max }\right)-\mathbf{x}^{\text {final }}=0
\end{aligned}
$$

Here $\mathbf{x}_{L T 1}$ is the front medial point of the left foot and $\mathbf{x}^{\text {init }}$ and $\mathbf{x}^{\text {final }}$ are its respective initial and final locations.

\subsubsection{Static initial / final conditions (SIF)}

In the current work, it is desired that the model begin from a static initial position and end at a static final position. To achieve this, the following additional equality conditions are imposed to set the first and second derivatives of all the degrees of freedom to be zero at initial and final time frames:

$$
\begin{aligned}
& h_{k}^{\text {sif }}(\boldsymbol{\Theta})=\dot{q}_{k}\left(\boldsymbol{\Theta}, t_{\min }\right)=0 \\
& h_{k+n_{\text {dof }}}^{\text {sif }}(\boldsymbol{\Theta})=\ddot{q}_{k}\left(\boldsymbol{\Theta}, t_{\min }\right)=0 \\
& h_{k+2 n_{\text {dof }}}^{\text {sif }}(\boldsymbol{\Theta})=\ddot{q}_{k}\left(\boldsymbol{\Theta}, t_{\max }\right)=0 \\
& h_{k+3 n_{\text {dof }}}^{\text {sif }}(\boldsymbol{\Theta})=\ddot{q}_{k}\left(\boldsymbol{\Theta}, t_{\max }\right)=0
\end{aligned}
$$

\subsubsection{Zero yawing moment (ZYM)}

As discussed in Section 2.1.1, there are two major forces that act on the walking biped, the inertial and gravity force (IGF) and the ground reaction force (GRF). The yaw component of moment due to the GRF is negligible relative to that of the IGF. To avoid excessive yawing motion of the human model, the alternating arm-leg motions should by themselves create a nearly vanishing resultant yawing moment by the IGF. That is, for example, the swinging-forward of left leg occurs concurrently with the swing-forward of the right arm and swing-backward of the left arm so that yawing moment by leg motion cancels out that by the arm motion. Such motions that yield self-equilibration of the yawing moment are achieved herein by imposing the following inequality condition on the model: 


$$
g^{\text {zym }}(\boldsymbol{\Theta}, t)=Y_{D}^{I G F^{2}}-Y^{\max ^{2}} \leq 0
$$

where

$$
Y_{D}^{I G F}=\mathbf{M}_{D}^{I G F} \cdot \mathbf{n}=\sum_{i}^{n_{\text {body }}}\left[\mathbf{x}_{D G i} \times\left(m_{i} \mathbf{g}-m_{i} \ddot{\mathbf{x}}_{G i}\right)\right] \cdot \mathbf{n} .
$$

Above, $Y_{D}^{I G F}$ is the resultant yawing component (normal to the ground) of IGF moment about the ZMP and $Y^{\max }$ is a specified upper bound on the magnitude of the yawing moment taken to be very small $(=0.01 \mathrm{~N} \cdot \mathrm{m})$ in this study. Also, $n_{\text {body }}$ denotes the number of body segments that comprise the model.

\subsubsection{Friction force limit (FFL)}

Since it is assumed in this study that the human model walks on a frictional surface, the in-plane magnitude of the ground reaction force at any instant is bounded above by the product of the normal GRF and a coefficient of friction. Because the in-plane magnitude of the IGF is equal and opposite to that of the GRF (by equilibrium of linear forces on the biped) the constraint can actually be applied to the in-plane magnitude of the IGF. The condition is thus imposed by the following inequality constraint:

$$
g^{\text {fll }}(\Theta, t)=\left(F_{\text {fric }}^{I G F}\right)^{2}-\mu^{2}\left(F_{\text {norm }}^{I G F}\right)^{2} \leq 0
$$

where

$$
F_{\text {fric }}^{I G F}=\left\|\mathbf{F}^{I G F} \times \mathbf{n}\right\| \text { and } F_{\text {norm }}^{I G F}=\mathbf{F}^{I G F} \cdot \mathbf{n} .
$$

Above, $\mathbf{F}^{I G F}$ is the IGF and $\mu$ is the friction coefficient assumed to be 0.45 in this study.

\subsection{CONTINUITY ISSUES FOR CONSTRAINT FUNCTION GRADIENTS AND ADDITIONAL CONSTRAINTS}

Gradient-based optimization methods find optimal solutions by iteratively: (1) assessing optimality at a trial solution point by considering cost/constraint function values and their gradients; and (2) searching for a subsequent trial solution point if the current point is not optimal. If cost/constraint functions and/or their gradients are discontinuous, the iterative optimization process may experience difficulty in converging to an optimal solution.

A representative functional that features potential discontinuity is one imposing a noslippage condition between the ground and foot contact points. The no slipping condition is that the tangential velocity to the ground plane of foot-point in ground contact must vanish. This is expressed as:

$$
\left\|\dot{\mathbf{x}}_{\eta}(\boldsymbol{\Theta}, t) \times \mathbf{n}\right\|=0, \quad \eta \in \text { footpoints in ground contact }
$$

where $\dot{\mathbf{x}}_{\eta}$ is the velocity of the foot-point $\eta$; and $\Theta$ is the set of design variables that include the control points for joint angle profiles and stage durations. For each foot-point, 
the no slipping condition has the following constraint with a switching condition that depends on the foot point height above the ground plane:

$$
g_{\eta}^{n s}(\boldsymbol{\Theta}, t)=\left[\begin{array}{lr}
0 & \left(\mathbf{x}_{\eta}(\boldsymbol{\Theta}, t) \cdot \mathbf{n}>0\right) \\
\left\|\dot{\mathbf{x}}_{\eta}(\boldsymbol{\Theta}, t) \times \mathbf{n}\right\| & \left(\mathbf{x}_{\eta}(\boldsymbol{\Theta}, t) \cdot \mathbf{n}=0\right)
\end{array}\right.
$$

Only a special mechanism such as a rolling wheel can satisfy this condition since the contact point between the wheel and ground is an instantaneous center of rotation with no slippage. Most other mechanisms will not satisfy the condition and thus both the functional and its gradient with respect to the design variables become discontinuous. To avoid the potential functional discontinuity, two alternative methods are applied in this study: 1) an artificial constraint of step control is imposed on the problem; and 2) locally-uniform time discretization is applied. Both measures impose additional constraints on the optimization problem beyond those introduced in the Sec 3.2.

\subsubsection{Stepping pattern control}

Stepping pattern control is to specify: 1) the number of gait stages and 2) the sequence of stepping patterns of corresponding gait stages. For example, consider two-step gait motion whose sequence of foot support conditions is LDS-LSS-RDS-RSS-LDS. If each gait stage's motion is represented by a single time segment bounded by two consecutive knots $\left[t_{i}, t_{i+1}\right)$, then the set of foot-points in ground contact during this stage $\Psi_{c}(t, \mathbf{t})$ can be given in mathematical form as:

$$
\begin{aligned}
& \Psi_{c}(t, \mathbf{t})=\left\{\eta \mid \mathbf{x}_{\eta}(\boldsymbol{\Theta}, t) \cdot \mathbf{n}=0 \text { for } t \in\left[t_{i}, t_{i+1}\right)\right\} \\
& \Psi_{f}(t, \mathbf{t})=\Psi_{c}^{C}
\end{aligned}
$$

Note that for a given $\mathrm{t}, \Psi_{c}(t, \mathbf{t})$ varies with the distribution of knots $\mathbf{t}$ since the gait stage or time segment that incorporates $t$ varies with the knot distribution. Above, $\Psi_{f}$ represents the complementary set of foot points that are not in ground contact.

\subsubsection{Locally-uniform sample time frames}

For motion planning optimization, constraint functions are evaluated at discrete sample times from each time segment. In this study, sample times are uniformly distributed within each time segment. For example the set of $n_{\text {samp }}$ time sample points on the $\mathrm{i}^{\text {th }}$ time segment are as follows:

$$
s_{i p}=p \frac{\left(t_{i+1}-t_{i}\right)}{n_{\text {samp }}}+t_{i} \quad\left[p=0,1, \ldots,\left(n_{\text {samp }}-1\right)\right]
$$

A sample time $s_{i p}$ always belongs to the $\mathrm{i}^{\text {th }}$ time segment, even with variation in the distribution of knots. Accordingly, the set of foot-points in ground contact $\Psi_{c}\left(s_{i p}\right)$ at sample time $s_{i p}$ is not a function of design variables. Tying this back in to the original issue, the non-slippage constraint function of Eq. (40) can now be rewritten as: 


$$
g_{\eta}^{n s}\left(\eta, s_{i p}\right)=\left[\begin{array}{ll}
0 & \left(\eta \in \Psi_{f}\left(s_{i p}\right)\right) \\
\left\|\dot{\mathbf{x}}_{\eta}\left(\boldsymbol{\Theta}, s_{i p}\right) \times \mathbf{n}\right\|=0 & \left(\eta \in \Psi_{c}\left(s_{i p}\right)\right)
\end{array}\right.
$$

Now the no slipping condition in Eq. (41) switches only on foot-point $\eta$ and sample time $s_{i p}$, and thus it does not switch with normal distance of foot-point to the ground that is a function of design variables $\Theta$ as in Eq. (38). Accordingly its gradient in the space of design variables $\Theta$ becomes continuous.

\section{NUMERICAL EXAMPLES}

The proposed motion planning method is tested here on a simplified virtual human modeled as a multi-linkage system with 25 degrees of freedom for the relative joint angles $\left\{q_{3}, q_{4}, \ldots, q_{27}\right\}$ and 6 degrees of freedom for rigid body pelvis rotations $\left\{q_{0}, q_{1}, q_{2}\right\}$ and pelvis translations $\left\{q_{28}, q_{29}, q_{30}\right\}$. The dimensions and degrees of freedom of the virtual human model are shown in Fig. 4. Since the framework is based on gradient-based optimization with inverse dynamics, the specific cost and constraint functions and design variables utilized to achieve somewhat realistic normal walking motions with the model are described. Three numerical examples of a biped walking on a level plane, and ascending on gently and steep inclined planes are presented (Fig. 6).

\subsection{Walking on a Level Plane}

To realize somewhat normal walking of the virtual human model, the cost or objective function chosen for minimization is deviation of the trunk from an upright posture. The constraints imposed are: (1) the virtual human takes three steps with the corresponding foot-support sequence: LDS-LSS-RDS-RSS-LDS-LSS-RDS; (2) The walking distance is one meter with $\mathbf{x}^{\text {init }}=(-0.5,0,0)$ and $\mathbf{x}^{\text {final }}=(0.5,0,0)$; (3) There is no joint velocity or acceleration at the beginning and end of motion. Additional constraints for dynamic equilibrium, joint angle limits, foot collision avoidance, zero yawing moment, friction force limit conditions are also imposed throughout the motion. Other walking parameters such as intermediate supporting foot locations, the time durations for single support and double support phases are not specified, but are instead optimized. The flexibility to optimize such walking parameters is one of the crucial factors of the proposed motion planning methods that permits adaptability. The number of time segments is limited to one per gait stage to minimize the optimization problem size. The cost and constraint functions are evaluated at six sample time points per time segment.

The actual optimization problems associated with the motion planning are solved here with SNOPT ${ }^{\mathrm{TM}}$ that employs an SQP (sequential quadratic programming) algorithm specialized for large-scale constrained optimization problems with smooth nonlinear cost and constraint functions. As in Eq. (26), inequality constraints are treated as equality constraints with the sum of their squared violations integrated over time to reduce the number of constraints. Motion planning of the three step normal walking on a level plane here took a total of 58 major iterations to converge, and required only 840 cpu-seconds on a Pentium ${ }^{(R)} 43.60 \mathrm{GHz}$ computer. By far, most of the cpu-time consumed was dedicated to information transfer back and forth between the optimization program and the motion planning software that computed all functionals 
and their gradients. Of the 840 seconds of total cpu-time, the actual optimization operations consumed only approximately four (4) seconds.

slope

Figure 6. Locomotion of a biped which demonstrates normal walking on a level plane $(a-b)$; on a gently inclined plane (c-d); and on a steeply inclined plane (e-f). To facilitate viewing, the posture has been offset walking direction. 


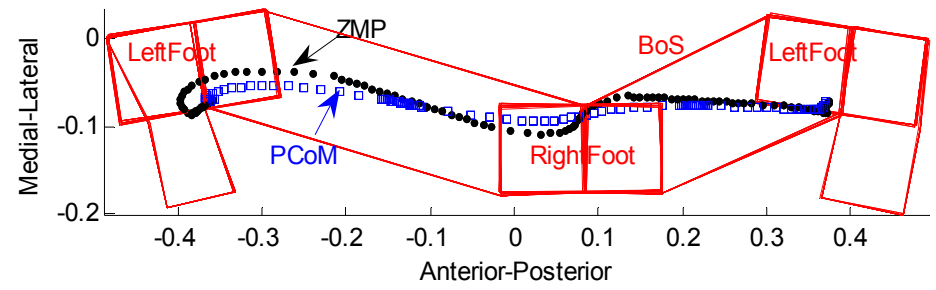

Figure 7. The trajectories for the base of support (BoS), projected center of mass (PCoM), and zero moment point (ZMP) for normal walking on a level plane. The ZMP (black dots) and PCoM (blue open squares) traverse the BoS (red outline)

The optimal walking locomotion for the model utilized is shown in Fig. 6 from different viewpoints with the postures shown taken at intervals of about 0.5 seconds. As would be expected, the modeled walking shows knee-bending of the swinging leg to avoid foot-collision with the ground and alternating arm-leg motion to cancel out the yawing moment created by the inertia forces from the swinging leg. The trajectories of the ZMP and the projected center of mass (PCoM) are shown in Figure 7 relative to supporting footprints or the base of support (BoS). In the figure, both the ZMP and the PCoM remain close to the imaginary centerline curve traced out by medial supporting foot-points. Although these spatial trajectories overlap each other, they feature relative lead-lag motion in the anterior-posterior direction (Fig. 8.a) although not in the medial direction (8.b). In either direction, the ZMP always remains within the instantaneous BoS to maintain dynamic equilibrium. The PCoM, however, traces a simpler curve in time that reflects steady motion of the trunk that carries most of the body mass and sometimes strays outside of the instantaneous BoS. 


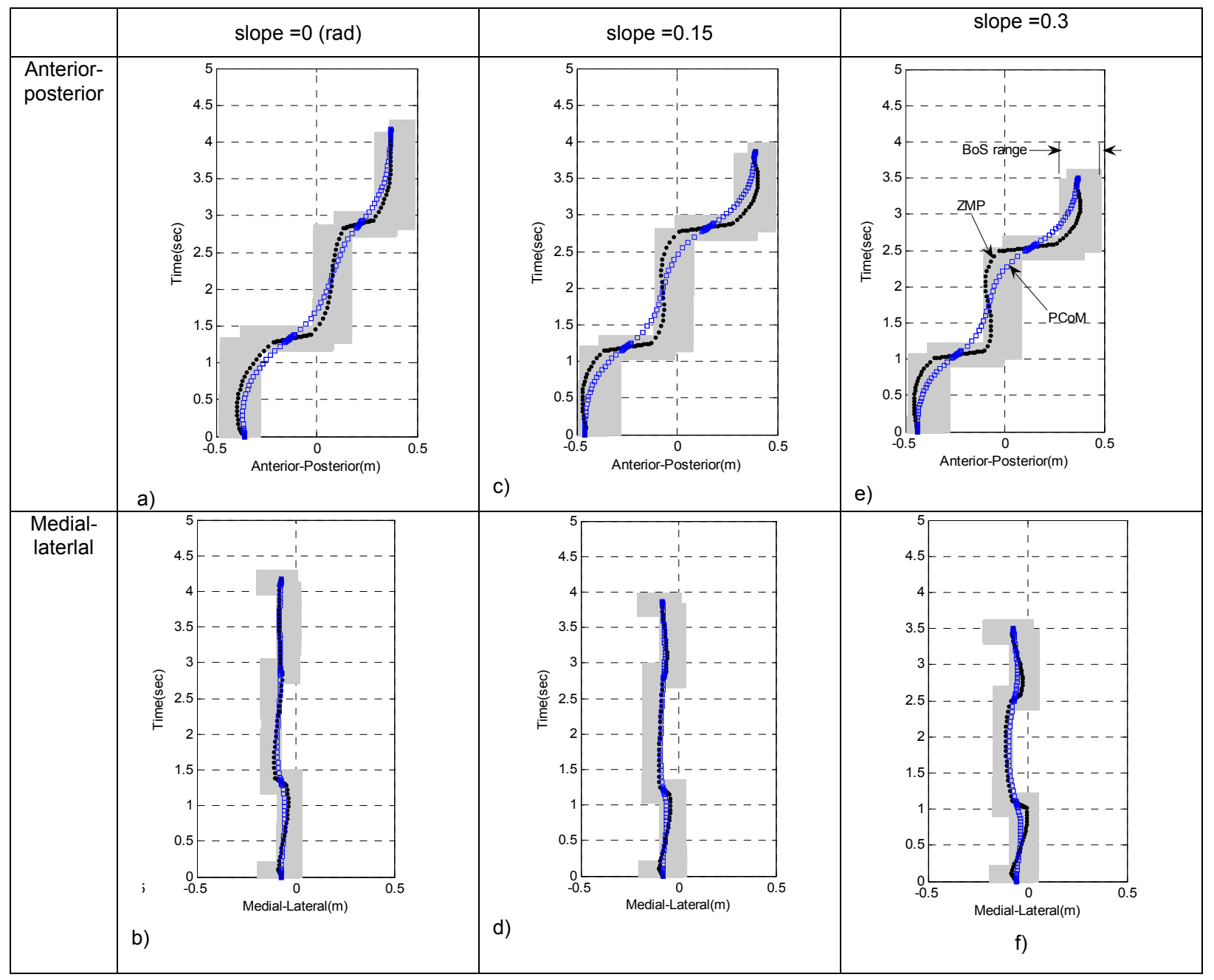

Figure 8. ZMP (black filled dots) and PCoM (blue open squares) trajectories over the BoS (gray regions) in anterior-posterior direction $(a, c, e)$, and in medial-lateral direction $(b, d, f)$ for normal walking on a level plane, walking on a plane sloped at 0.15 , and walking on a plane sloped at 0.30 , respectively. 
Further elaboration on the movement of the ZMP in time is shown in Figures 9 and 10. The gait STAGE 3 (Fig. 9) involves double support with the right foot out in front (RDS). During this stage the ZMP makes substantial forward progress and moves ahead of the PCoM as the weight is transferred from the left foot to the right. During the subsequent motions of STAGE 4 (Fig. 10) which involves right foot single support (RSS) with the forward swinging of the left leg, the ZMP falls back behind the PCoM.

As noted previously, the durations of the seven gait stages modeled herein are not prescribed and are determined as part of the modeling problem. The optimal stage durations computed for the problem at hand are shown in Fig. 11. The sum of the stage durations is the total travel time of $4.65 \mathrm{sec}$. The odd stage durations $(1,3,5,7)$ in Fig. 11 correspond to double support conditions and the even stages $(2,4,6)$ to single support. It is noteworthy that the durations for all the double support stages are 0.1 second, which is the minimum allowed.

The need to specify minimum durations for the double foot support stages requires some explanation. There are two primary reasons for imposing minimum durations of the stages. The first is that this precludes singularity of the modified B-Spline basis functions in Eq. (14). Secondly, as pointed out in discussion of Fig. 10, the ZMP makes significant forward progress during the double foot support stages and even moves well ahead of the PCoM, albeit temporarily. The reason for such separation developing between the ZMP and PCoM is the significant inertia forces associated with the body's forward motion. One way to generate sufficient inertia forces with both feet on the ground and the trunk upright is to minimize the stage duration. Note that if the stage duration is reduced by a factor of two, the magnitude of inertial forces increase by the factor of four with same range of motion. Due to this tendency for the double-foot support stages to be very short, and potential singularity of the spline basis functions as the durations tend to zero, lower bounds on these stage durations are imposed.

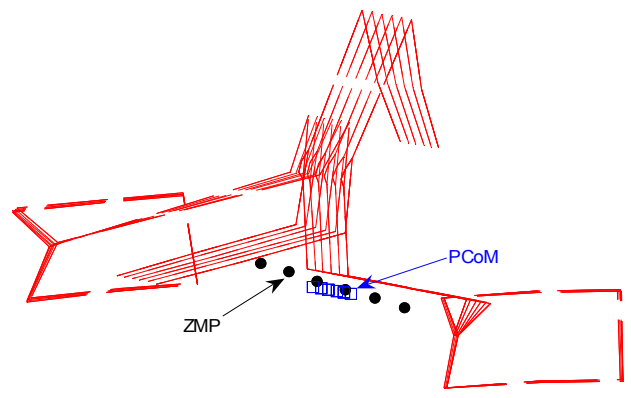

a) b)

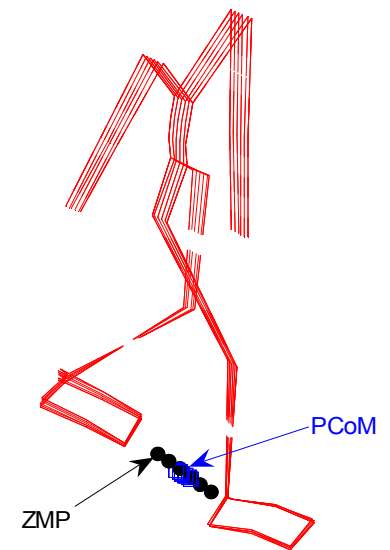

Figure 9. STAGE 3 locomotion during double support phase with right foot in the front (RDS) : (a) view from top and (b) side view from right. The figures in dashed-red lines denote the array of body postures; the six black dots trace out the ZMP trajectory, and blue open squares the PCoM trajectory. 


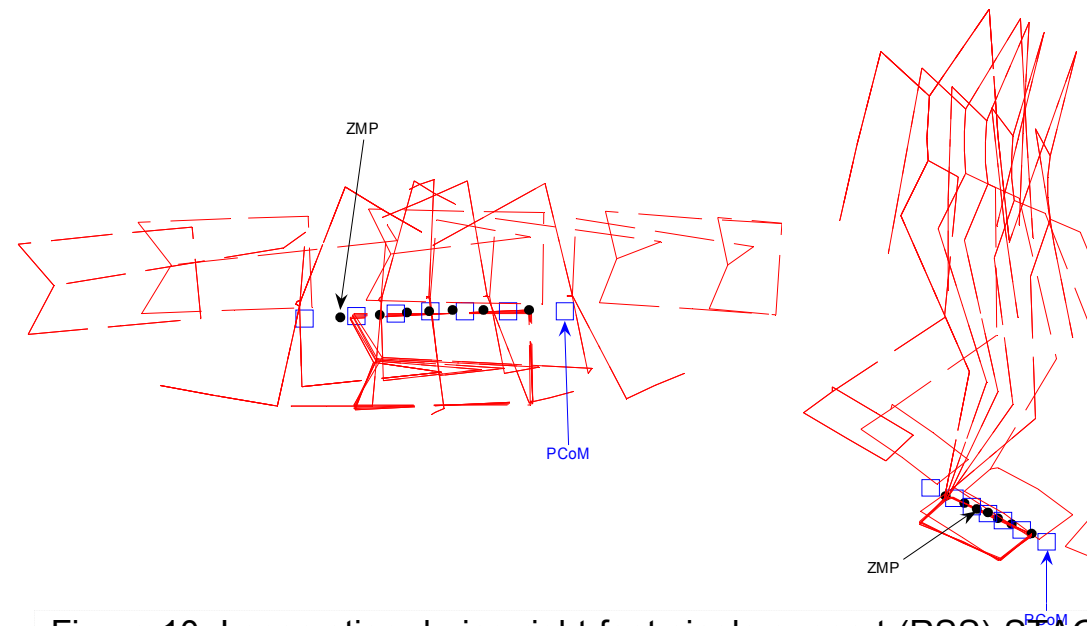

Figure 10. Locomotion during right foot single support (RSS) STA ÁGE 4 as the left leg swings forward: (a) view from top and (b) side view from right. The figures in dashed-red lines again denote the array of the postures while the black filled dots show the ZMP trajectory, and the blue open squares the PCoM.

The time histories of right hip extension $\left(q_{13}\right)$, right knee extension $\left(q_{14}\right)$ and right ankle plantar flexion $\left(q_{15}\right)$ are representative joint angles in the human model and are plotted in Fig. 12. Their first and second time derivatives are also shown. Since the joint angle histories in time are interpolated using linear combinations of cubic B-spline basis functions, the second time derivatives of the joint angle histories are continuous but feature piecewise linear behavior. In Fig. 12, the first and second time derivatives are scaled to fit onto the same axes as the joint angle histories.

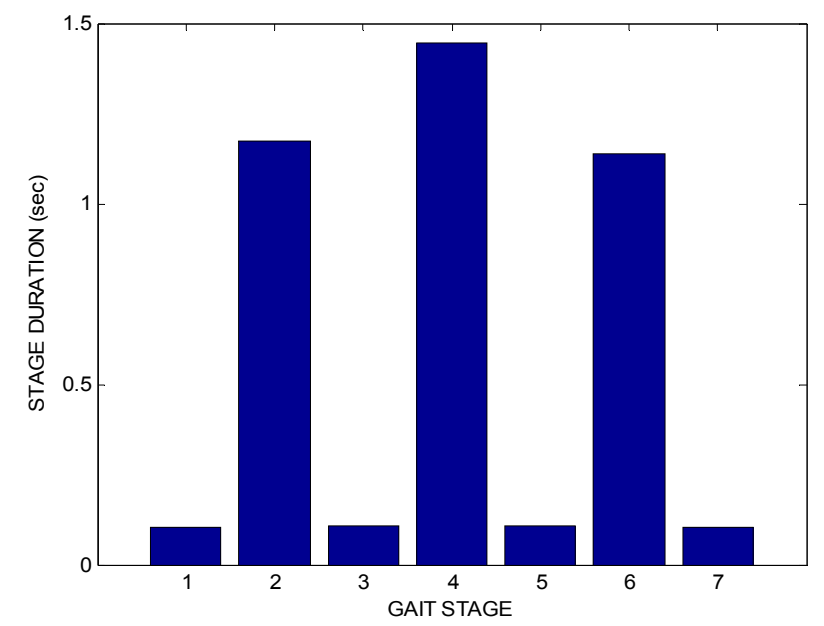

Figure 11. Computed gait stage durations in seconds for normal walking on a level plane. 


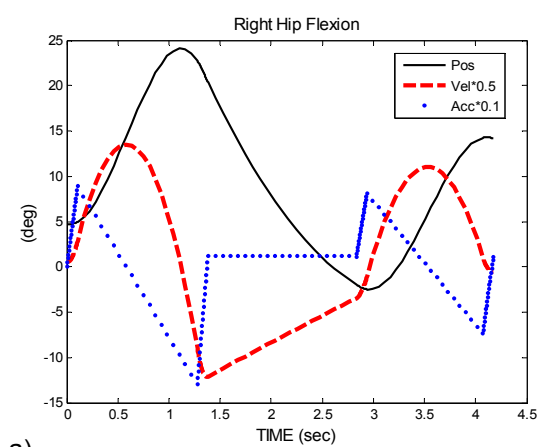

a)

Figure 12. Profiles of joint angles for: a) hip flexion, b) knee extension and c) ankle dorsiflexion. Both first and second time derivatives of the joint angles are also shown to illustrate their continuity characteristics. The time derivatives are scaled as noted to fit onto their angle rotation graphs.

\subsection{Slope ascending}

Two examples of the biped model ascending an inclined plane are also presented here. In the first, the plane is inclined at a slope of 0.15 with respect to the horizontal, and in the second the slope magnitude is 0.30 . For comparison purposes, all the parameters except for the gravity vector are kept same in these simulations as they were for normal walking on a level plane. When the ground plane is inclined with angle $\alpha$ in the human model's sagittal plane, the gravity vector is divided into two components in terms of axes attached to the slope (Fig. 12):

$$
\mathbf{g}(\alpha)=C_{g}\left(\sin (\alpha) \mathbf{e}_{x}+\cos (\alpha) \mathbf{e}_{z}\right)
$$

where $C_{g}\left(=-9.81 \mathrm{~m} / \mathrm{s}^{2}\right)$ is a gravity constant; $\mathbf{e}_{x}$ is a unit vector in the anterior direction on the slope; and $\mathbf{e}_{z}$ the unit vector normal direction to the slope. In essence, the slope ascending problem is very much like walking on a level plane with the exception that now the posterior gravity force $\left(=C_{g} \sin (\alpha) \mathbf{e}_{x}\right)$ constantly pulls the biped back in the posterior direction throughout the motion (Fig 14.b) and its normal component $\left(=C_{g} \cos (\alpha) \mathbf{e}_{z}\right)$ is reduced. The cost function for upright trunk posture measure is generic enough to be applied to slope ascent motion planning, in that the minimum trunk deviation is achieved by aligning the trunk to the gravity vector [Eq. 27]. 


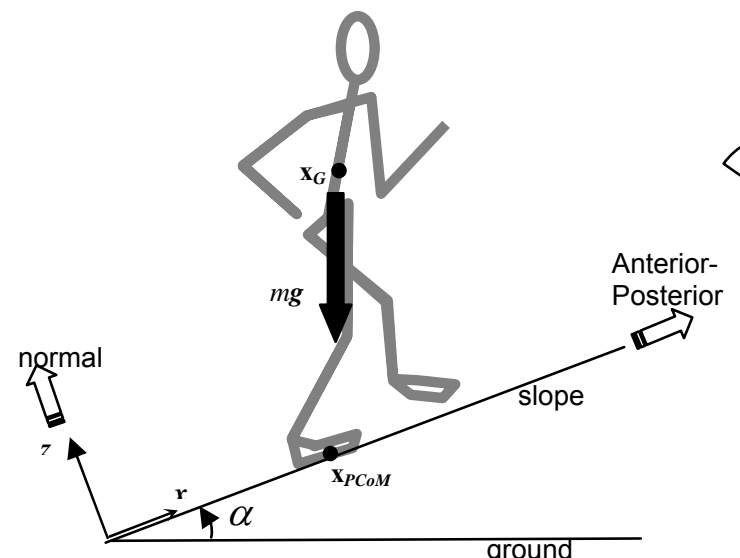

a)
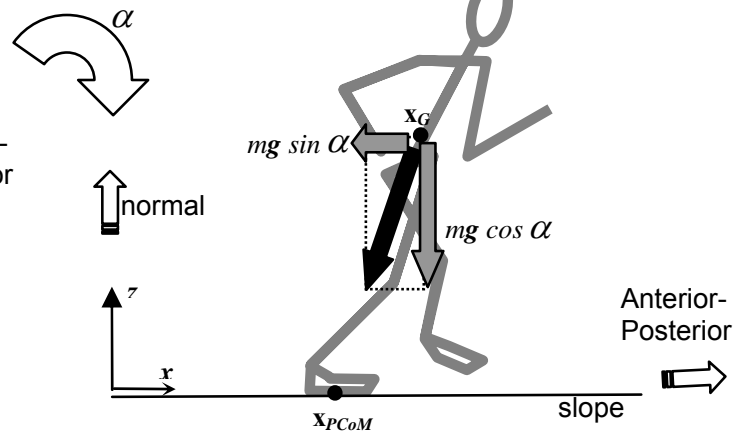

b)

Fig 13. Biped model's ascent of an inclined slope: a) xyz-axes are attached to slope; b) when the system is rotated by $\alpha$, the biped ascent is equivalent to normal walking with fraction of gravity force $(m g \sin \alpha)$ that pulls the biped backward.

The optimal slope ascent locomotions are shown in Figs. 6.c-d for the gentle slope and Figs. 6.e-f for the steeper slope. In the noted figures, the $x y z$-axes are rotated from level ground back to original inclined slope for rendering. The initial and final postures show forward leaning trunk postures to keep the static equilibrium imposed by the static constraints (Eq. 32) at the initial and final postures. By leaning forward the biped generates positive tipping moment about $y$-axis to counter the opposing moment by the posterior gravity force. While moving, the biped can generate moment to counteract the posterior gravity moment with the inertial forces from limb and trunk movement. Such non-zero inertial forces result in separation of the ZMP and PCoM during motion while zero inertial forces result in their coincidence at the initial and final frames when static equilibrium is imposed (Fig. 8). As the slope gets steeper, larger inertia forces are needed, and these are achieved in the model by reducing the total travel time. The cost function values increase significantly with increase in steepness of the slope. The number of optimization iterations and cost function values for all three walking cases are shown in Table 3 and these indicate that as the slope steepness increases, the number of optimization iterations and cpu-time required to solve the problem increase.

Table 3. Cost function values, cpu execution times, and required optimization iterations for different cases of biped walking.

\begin{tabular}{|c|c|c|c|}
\hline & Cost & CPU time (sec) & Iterations [minor] \\
\hline $\begin{array}{c}\text { Normal walking } \\
\text { [level plane] }\end{array}$ & $3.80 \times 10^{-4}$ & 840 & $58[464]$ \\
\hline $\begin{array}{c}\text { Slope ascent } \\
\text { [slope }=0.15]\end{array}$ & $2.16 \times 10^{-3}$ & 1260 & $87[483]$ \\
\hline $\begin{array}{c}\text { Slope ascent } \\
\text { [slope=0.30] }\end{array}$ & $5.37 \times 10^{-3}$ & 1380 & $92[625]$ \\
\hline
\end{tabular}




\section{DISCUSSION AND CONCLUSIONS}

The proposed ZMP-based formulation for motion planning associated with bipedal locomotion satisfies the unilaterality condition with unknown ground reaction forces as well as dynamic equilibrium conditions without the necessity of quantifying actuating joint torques. The formulation is highly efficient in that it avoids the time-consuming expense associated with having to solve equations of motion over the time frame of interest. The method poses motion planning associated with walking as an optimization problem. In the example problems presented all of the constraints were aggregated into a single equality constraint. This treatment of constraints led to rapid convergence to feasible and optimal solutions in all three of the examples presented.

Although the proposed formulation does not require solving for joint actuating torques, it must be employed with caution. Since ZMP-based dynamic equilibrium conditions take care of only two in-plane angular components of resultant forces (cf. Eq. 5), additional constraints for equilibrium are required for completeness: one for outof-plane components (i.e. yawing moment) and another for each of the linear components (i.e. two in-plane and one normal forces). In the current formulation, therefore, the magnitude of yawing moment by inertia and gravity forces is assumed to vanish since the yawing moment produced by the frictional components of the groundreaction forces have a very small moment arm (less than the foot-length dimensions) about the center of pressure. With yawing moments associated with the ground-reaction forces very small, the yawing moments due to inertia and gravity forces should equilibrate themselves. Also, a physical constraint that restricts the magnitude of friction forces to be only a fraction of the normal ground reaction force should be properly applied to three linear components of inertial forces (Eq. 36).

Since the ZMP-based formulation explained and demonstrated herein does not involve joint actuating torques, it is hard to impose any constraints or performance measures associated with actuating torques. Nevertheless, even in its current form, the ZMP-based formulation can still be an extremely valuable tool for exploring changes in walking parameters (i.e. step length, cadence, stage durations, foot placement, etc) that keep walking stability.

When inverse dynamics is used for motion planning, the smoothness in time of the assumed displacement field acts as an implicit constraint that could perhaps conflict with other constraints and may make the solution domain infeasible. In the proposed methods, the number of control points for cubic B-spline curve approximation is the control parameter for the smoothness constraint. But, there is no general way of knowing the minimum number of control points necessary to secure the feasibility of the problem. Though more control points can increase the smoothness of displacement field and can secure feasibility of the problem, the computational cost increases significantly with it. When more artificial constraints (i.e. to specify travel time, initial posture or PCoM trajectory) come into consideration, it increases the potential conflict with the smoothness constraint. Thus, by using a minimum number of artificial constraints, the proposed optimization formulation can secure the feasible domain and minimize the computational cost with small number of control points. 
The motion planning formulation was exercised on three different problems involving walking on a level plane, walking on a gently sloped plane, and walking on a more steeply inclined plane. These problems were solved in between 840 and $1380 \mathrm{cpu}-$ seconds on a current generation personal computer. As the slope became steeper, more iterations were required to obtain feasible motion-planning solutions, and consequently the cpu-time required also increased. The cost or objection function values associated with walking up an inclined plane were also considerably higher than that for walking on a level plane. These results would be consistent with the observation that as the feasible domain of a motion planning problem becomes more restricted, (as is the case for walking up a sloped plane), one can anticipate a higher optimal cost value and more optimization iterations to find the optimum.

The authors believe that while the computational times reported here for the motionplanning computations are quite small (i.e. between 14 and 22 cpu-minutes), the times can be still be reduced by approximately an order of magnitude by keeping both the optimizer and the motion-planning software in the computer's shared RAM simultaneously. This would significantly reduce the time required for information transfer (functional values and their gradients) between the processes.

There are a multitude of potential applications for modeling of human walking as described herein. From a biomechanics perspective, a first goal is to better understand normal locomotion in healthy individuals and to validate the framework's ability to predict the gaits that constitute normal locomotion. If this can be achieved with a high degree of confidence, then such models might then be turned to study and address locomotion in humans with gait pathologies stemming from a wide range of causal mechanisms. While the model has been exercised here to explore normal walking motions, it is foreseen that the model might with further development be a very useful in exploring how limb or skeletal asymmetries affect or create abnormal locomotion. The formulation might further be used to devise intervention techniques that would help to restore normal locomotion to patients.

\section{ACKNOWLEDGMENTS}

This research is funded by the US Army TACOM project: Digital Humans and Virtual Reality for Future Combat Systems (FCS) (Contract No.: DAAE07-03-Q-BAA1).

\section{REFERENCES}

Anderson, F. C. and Pandy, M. G. (2001). "Dynamic optimization of human walking." Journal of Biomechanical Engineering, v 123, 5, 381-390.

Azevedo, C., Poignet, P. and Espiau, B. (2002). "Moving horizon control for biped robots without reference trajectory." Proceedings of the 2002 IEEE, International Conference on Robotics \& Automation, Washington, DC, 2762-2767.

Bessonnet, G., Sardain, P. and Chesse, S. (2002). "Optimal motion synthesis- dynamic modeling and numerical solving aspects." Multibody System Dynamics, 8(3), 257-278. 
Chevallereau, C.; Formal'sky, A. and Perrin, B. (1998). "Low energy cost reference trajectories for a biped robot." Proceedings of IEEE International Conference on Robotics and Automation. (held in Leuven, Belgium), 3, 1398-1403.

Chevallereau, C. and Aousin, Y. (2001). "Optimal reference trajectories for walking and running of a biped robot." Robotica, 19, 557-569.

Denavit, J. and Hartenberg, R. S. (1955). "A kinematic notation for lower-pair mechanisms based on matrices." ASME Journal of Applied Mechanics, 22, 215-221.

Faraway, J. J., Zhang, X. and Chaffin, D. B. (1999). "Rectifying postures reconstructed from joint angles to meet constraints." Journal of Biomechanics. 32 (7), 7333-736.

Featherstone, R. Robot Dynamics Algorithms. Kluwer Academic Publishers,Norwell, Massachusetts, 1987.

Furusho, J. and Masubuchi, M. (1986). "Control of a dynamical biped locomotion system for steady walking. Journal of Dynamic System, Measurement, and Control. $108,111-118$.

Gill, P. E., Murray, W. and Saunders, M. A. (2002). "SNOPT: An SQP algorithm for large-scale constrained optimization." SIAM J. Optim. 12, 979-1006.

Goswami, A. (1999). "Postural stability of biped robots and the foot rotation indicator point." International Journal of Robotics Research, 18(6), 523-533.

Kuffner, J., James, J., Kagami, S., Nishiwaki, K. Inaba, M. and Inoe, H. (2002). "Dynamically-stable motion prediction for humanoid robots." Autonomous Robots, 12, 105-118.

Lo, J., Huang, G. and Metaxas, D. (2002). "Human motion prediction based on recursive dynamics and optimal control techniques." Multibody System Dynamics, 8, 433-458.

Nagasaka, K., Inoue, H. and Inaba, M. (1999). "Dynamic walking pattern generation for a humanoid robot." Proceedings of the IEEE International Conference on Systems, Man and Cybernetics, 6, VI-908 - VI-913.

Nishiwaki, K. and Kagami, S. (2002). "Online generation of humanoid walking motion based on a fast generation method of motion pattern that follows desired ZMP." IEEE International Conference on Intelligent Robots and Systems, 3, 2684-2689.

Pandy, M. G., Anderson, F. C. and Hull, D. G. (1992). "A parameter optimization approach for the optimal control of large-scale musculoskeletal systems." J. of Biomechanical Engineering, Transactions of the ASME, 114(4), 450-460.

Pandy, M. G. (2001). "Computer modeling and simulation of human movement." Ann. Rev. Biomed. Eng., 3, 245-273. 
Perry J., (1992). Gait analysis; normal and pathological function. Thorofare, N.J.: Slack.

Piegl, L. and Tiller, W. (1997). The NURBS Book, $2^{\text {nd }}$ Ed. Springer-Verlag.

Rostami, M. and Bessonnet, G. (2001). "Sagittal gait of a biped robot during the single support phase. Part2: optimal motion." Robotica, 19(3), 241-253.

Roussel, L., Canudas-de-Wit, C. and Gowami, A. (2001). "Generation of energy optimal complete gait cycles for biped robots." Proceedings of IEEE International Conference on Robotics and Automation. (held in Leuven, Belgium), 3, 2036-2041.

Skinner H.B. (2003). "Current Diagnosis \& Treatment in Orthopedics" $3^{\text {rd }}$ Ed., McGrawHill.

Saidouni, T. and Bessonnet, G. (2003). "Generating globally optimized sagittal gait cycles of a biped robot." Robotica, 21(2), 199-210.

Sardain, P. and Bessonnet, G. (2004). "Forces acting on a biped robot. Center of pressure - zero moment point." IEEE Transations on systems, man and cyberneticsPart A: Systems and Humans, 34(5), 630-637.

Saunders JB, Inman VT, Eberhart HD., (1953). "The major determinants in normal and pathological gait." JBJS, 35-A:543-58.

Sutherland D. (1988). Development of mature walking. Philadelphia: MacKeith Press.

Sutherland DH, Kaufman KR, Moitoza JR. (1994). "Kinematics of normal human walking," In: Rose J, Gamble JG, eds. Human walking, 2nd ed. Baltimore: Williams \& Wilkins, 2:23-45.

Tang Z, Zhou C, and Sun Z. (2003). "Trajectory planning for smooth transition of a biped robot," Proceedings 2003 ICRA. IEEE 2, 2455-2460

Vukobratović, M. and Borovac, B. (2004). "Zero-moment point - thirty five years of its life." International Journal of Humanoid Robotics, 1(1), 157-173.

Wang, C. Y. E., Bobrow, J. E. and Reinkensmeyer, D. J. (2001). "Swinging from the hip: use of dynamic motion optimization in the design of robotic gait rehabilitation." Proceedings 2001 ICRA. IEEE 2, 1433 - 1438.

Wang, Q., Xiang, Y.-J., Kim, H.-J., Arora, J. S. and Abdel-Malek, K. (2005). "Alternative formulations for optimization-based digital human motion prediction." Paper 2005-012691, 2005 SAE Digital Human Modeling for Design and Engineering Symposium, lowa City, lowa, June 14-16, 2005. 
Yamaguchi, G. T. and Zajac, F. E. (1990). "Restoring unassisted natural gait to paraplegics via functional neuromuscular stimulation: a computer simulation study." IEEE Trans. Biomed. Eng., 37, 886-902. 